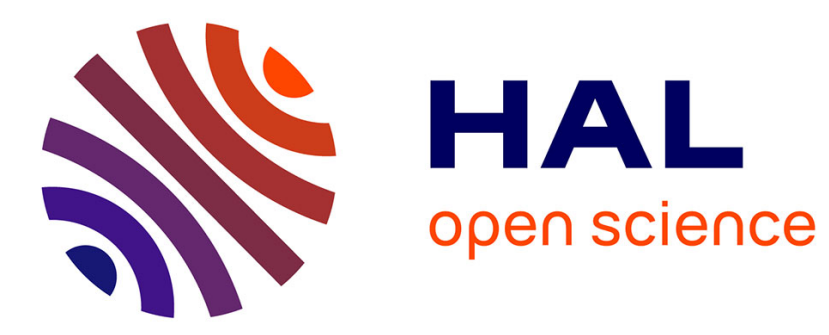

\title{
Application of aptamers for in vivo Molecular Imaging and Theranostics
}

Alix Bouvier-Müller, Frederic Duconge

\section{To cite this version:}

Alix Bouvier-Müller, Frederic Duconge. Application of aptamers for in vivo Molecular Imaging and Theranostics. Advanced Drug Delivery Reviews, 2018, 10.1016/j.addr.2018.08.004 . hal-02006476

\section{HAL Id: hal-02006476 \\ https://hal.science/hal-02006476}

Submitted on 4 Feb 2019

HAL is a multi-disciplinary open access archive for the deposit and dissemination of scientific research documents, whether they are published or not. The documents may come from teaching and research institutions in France or abroad, or from public or private research centers.
L'archive ouverte pluridisciplinaire HAL, est destinée au dépôt et à la diffusion de documents scientifiques de niveau recherche, publiés ou non, émanant des établissements d'enseignement et de recherche français ou étrangers, des laboratoires publics ou privés. 


\title{
Application of aptamers for in vivo Molecular Imaging and Theranostics.
}

\author{
Alix Bouvier-Müller ${ }^{1,2,3}$ and Frédéric Ducongé ${ }^{1,2,3 *}$ \\ ${ }^{1}$ CEA, Fundamental Research Division (DRF), Institut of Biology François Jacob (Jacob), Molecular \\ Imaging Research Center, 18 Route du Panorama, 92265 Fontenay-aux-Roses, France \\ ${ }^{2}$ Neurodegenerative Diseases Laboratory, CNRS CEA URA 2210, Fontenay-aux-Roses, France \\ ${ }^{3}$ Paris-Saclay University, Fontenay-aux-Roses, France
}

* Author to whom correspondence should be addressed; frederic.duconge@ cea.fr Tel.: +33-146-548641

\begin{abstract}
:
Nucleic acid aptamers are small three-dimensional structures of oligonucleotides selected to bind to a target of interest with high affinity and specificity. In vitro, aptamers already compete with antibodies to serve as imaging probes, e.g. for microscopy or flow cytometry. However, they are also increasingly used for in vivo molecular imaging. Accordingly, aptamers have been evaluated over the last twenty years in almost every imaging modality, including single photon emission computed tomography, positron emission tomography, magnetic resonance imaging, fluorescence imaging, echography, and x-ray computed tomography. This review focuses on the studies that were conducted in vivo with aptamer-based imaging probes. It also presents how aptamers have been recently used to develop new types of probes for multimodal imaging and theranostic applications.
\end{abstract}

Keywords: SELEX, in vivo imaging, imaging probes, nanomedicine, biodistribution 
Abbreviations:

BzdU: N-(benzylcarboxyamide)-2'-deoxyuridine

DiR: dioctadecyl-3,3,30,30-tetramethyl indotricarbocyanine iodide

EpCAM: epithelial cell adhesion molecule

LNA: locked nucleic acid

MRI: magnetic resonance imaging

MUC1: Mucin-1

NIR: near-infrared imaging

$\mathrm{NP}(\mathrm{s})$ : nanoparticle(s)

PDT: photodynamic therapy

PET: positron emission tomography

PSMA: prostate specific membrane antigen

PTT: photothermal therapy

SPECT: single photon emission computed tomography

SPION(s): superparamagnetic iron oxide nanocrystal(s)

X-ray CT: X - ray computerized tomography 


\section{Contents}

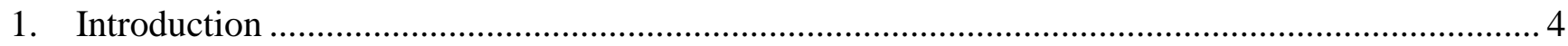

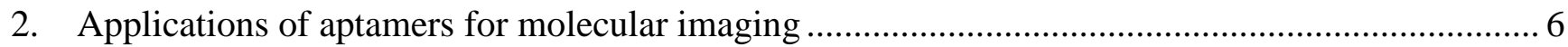

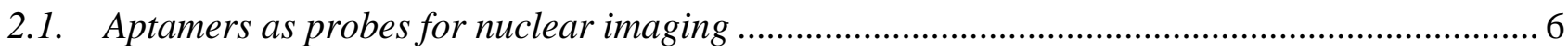

2.1.1 Single photon emission computed tomography (SPECT) .................................................. 6

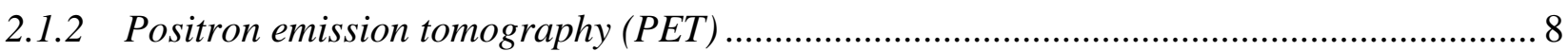

2.2. In vivo fluorescence imaging with aptamers .................................................................. 9

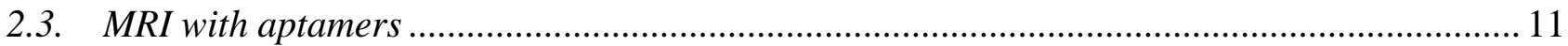

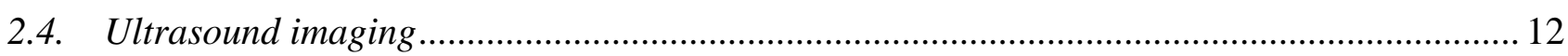

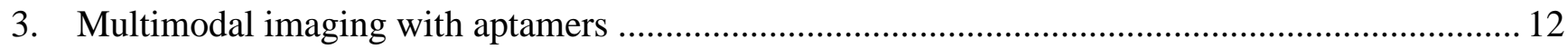

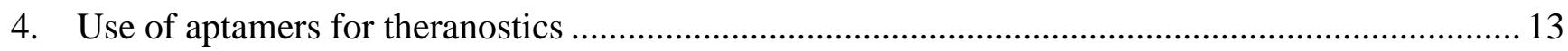

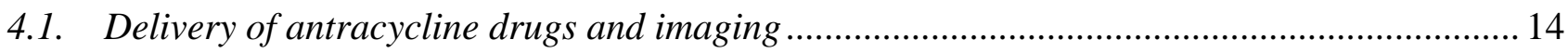

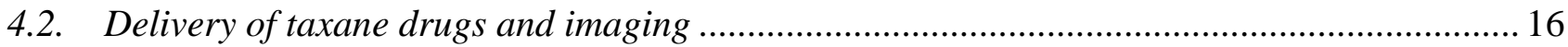

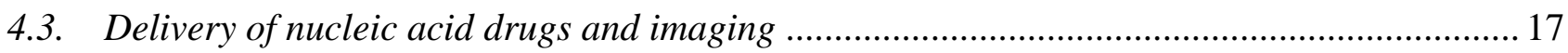

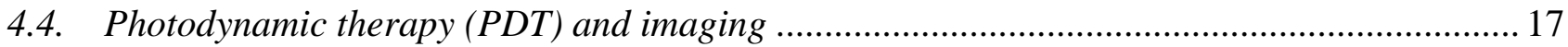

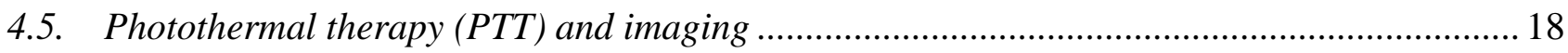

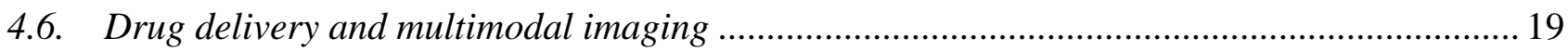

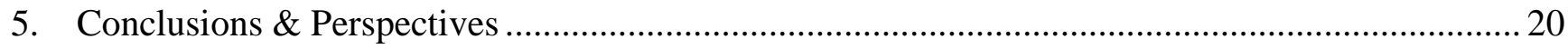

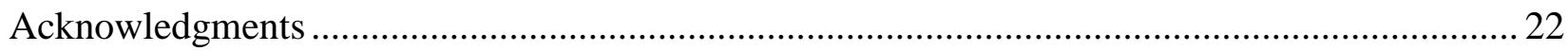

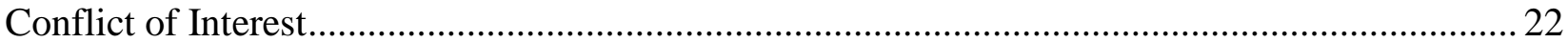

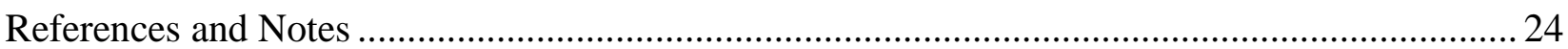




\section{Introduction}

Molecular imaging of living subjects allows the study of molecular and cellular events in their native environment, instead of having to remove cells or tissues before analysis. It is now routinely used in clinics to diagnose various diseases, as well as evaluate drug efficacy. There are several imaging modalities available and most require a molecular-imaging probe. Chemistry or biochemistry plays a crucial role in the development of such probes, which are usually constructed by combining three molecular components (Figure 1): 1) a component to provide a signal that can be detected by an imaging instrument, 2) a ligand that can specifically interact with the molecular target of interest, and 3) a spacer that links the two previous components together. The spacer is not always mandatory and the two first components may sometimes be directly linked together. Once introduced into the living subject, the location of the molecular imaging probes can be monitored by an imaging system. The probe is generally injected intravenously, but other routes of administration can be used. Just after injection, the probe is distributed throughout the body of the subject. Then, part of the probe is expected to bind to its molecular target, whereas the rest is cleared from the tissues. Accordingly, the imaging system should detect a higher signal in tissue expressing the target than the others (Figure 2).

The first molecular imaging probes were derived from natural molecules that are involved in metabolism. For example, one of the most famous radiotracers used for cancer imaging is 2-deoxy-2$\left[{ }^{18} \mathrm{~F}\right]$ fluoro-d-glucose $\left({ }^{18} \mathrm{~F}-\mathrm{FDG}\right)$, which is a radiolabeled glucose analog. However, there is now an increasing demand to develop new types of molecular imaging probes that can bind to targets for which there are no available or known natural ligands. These new probes are often made from macromolecules that can be tailored to interact with any molecular target of interest. These macromolecules use four forces of interaction (ionic bonds, hydrogen bonds, hydrophobic effects, and van der Waals interactions) to adopt three-dimensional (3D) structures and specifically interact with their target. Such forces are often called "weak bonds" because they require little energy to break. However, the 3D structure of a macromolecule can allow it to form many such weak interactions with a target of interest to, ultimately, bind to it with a very high affinity.

Peptides and proteins can provide a myriad of 3D structures able to generate high affinity ligands. For example, antibodies are well-known ligands that can bind to their target with very high affinity and specificity. This property has been exploited to develop drugs and molecular imaging probes. However, proteins have many disadvantages when used as molecular imaging probes. First, their 3D structures can be unstable and difficult to maintain, not only in vivo but also in vitro during labeling steps. In addition, they are almost impossible to produce chemically, except for small peptides. Thus, they require bio-production processes that are not only costly but can also cause problems of batch-to-batch reproducibility. Finally, they may face problems of immune response, e.g. 
only humanized antibodies can be injected into humans, but cannot be easily evaluated in immunocompetent animal models, which reject them.

Like amino-acid polymers, nucleic acid sequences can form many 3D structures. This property is used in nature by RNAs to interact with a variety of molecules or to have catalytic activities (e.g. protein synthesis or splicing). Since 1990, several combinatorial approaches have been developed to exploit and analyze these properties of nucleic acids. Tuerk and Gold popularized such methods as SELEX (for systematic evolution of ligands by exponential enrichment) [1] and the nucleic acid-based ligands identified by this technique were baptized "aptamers" by Ellington and Szostak from the Latin "aptus", meaning "to fit" [2]. Although the selection of aptamers has undergone many improvements and refinements [3-8], they are still based on the same principle (Figure. 3). A population of oligonucleotide sequences known as "candidates" is synthesized. They contain a randomly synthesized region, which is framed by two known sequences that can be amplified by PCR. This population is then subjected to an in vitro selection process, which extracts candidates that bind to a chosen target from the rest. These candidates are then amplified by PCR or RT-PCR and transcribed in vitro before being used in a new in vitro selection round. During the amplification step, mutations may occur, leading to the appearance of candidates that are slightly different from their parents, some of which may have higher affinity for the target. Accordingly, the population is expected to evolve through several rounds of selection and amplification, in a Darwinian fashion, favoring the progressive enrichment of the sequences with the highest affinity. Sequencing a sample of the population can then identify such sequences. Recently, high-throughput sequencing has led to a better understanding of this molecular evolution process and better identification of the aptamers [9]. The main weakness of RNA and DNA sequences is to be rapidly degraded by nucleases. The half-life of the oligonucleotide RNA is a few seconds in the plasma whereas for DNA it is around 30 to 60 minutes[10]. Several chemical modifications have been introduced in aptamers to protect them from nuclease degradation including modified bases, modified sugar and modifications on the phosphodiester linkage [11]. The most common strategy is to use chemically modified nucleotides that can be directly incorporated by polymerases during SELEX. For example, 2'Fluoro nucleotides are often used and the half-life time of 2'Fluro-pyrimidine RNA sequences is around 5 to 15 hours in plasma [10]. Chemical modifications could also be incorporated post-selection but they can change the structure of aptamers and affect their binding. Since their discovery, aptamers have been selected against a wide variety of targets, from small molecules to proteins to even complexes of targets present on the cell surface $[3,12,13]$. Aptamers have provided fundamental insights concerning the versatility of RNA, supporting its involvement in the origin of life $[14,15]$. In parallel, aptamers have been increasingly used for biotechnological applications, such as biosensors [16], bio-purification [17], biomarker discovery [18], 
and the regulation of gene expression [19]. Furthermore, one aptamer has already been commercialized as a drug for the treatment of age-related macular degeneration and several others are currently in clinical trials [20].

Because of their high specificity and affinity, aptamers are promising tools for molecular imaging [21-24]. Over the last 20 years, several aptamers have been tested as molecular probes in almost every imaging modality, including positron emission tomography (PET), single photon emission computed tomography (SPECT), magnetic resonance imaging (MRI), echography, X-ray computed tomography (X-ray CT), and fluorescence imaging. This review presents an up-to-date summary of the investigations that have been performed in vivo and the recent use of aptamers to develop new types of probes for multimodal imaging and theranostic applications. We have tried to exhaustively list all these studies in tables (see Tables 1, 2 and 3), but have chosen not to describe them all in detail, but rather to present general conclusions that can be drawn from these experiments.

\section{Applications of aptamers for molecular imaging}

\subsection{Aptamers as probes for nuclear imaging}

Nuclear imaging uses molecular imaging probes that are radioactively labeled. These probes are usually called "radiotracers" or "radiopharmaceuticals". Once injected into subjects, external detectors (gamma cameras) detect the radiation emitted by such probes. This signal is then used to reconstruct images that allow quantitative measures of probe localization at various times post-injection. Several different modalities of nuclear imaging can be used, depending on the radioisotope, some of which have been developed for the radiolabeling of aptamers [25, 26]. 2D scintigraphy or 3D imaging by SPECT use $\beta$ emitters (such as ${ }^{99 \mathrm{~m}} \mathrm{Tc},{ }^{123} \mathrm{I}$, or ${ }^{111} \mathrm{In}$ ) that can be directly detected by gamma cameras. In contrast, PET uses positron-emitting $\left(\beta^{+}\right)$radionuclides (such as ${ }^{11} \mathrm{C},{ }^{18} \mathrm{~F},{ }^{68} \mathrm{Ga}$, or ${ }^{64} \mathrm{Cu}$ ) that need first to be annihilated by an electron to release two gamma rays, which are emitted at an angle that approximates $180^{\circ}$. Radiolabeled aptamers have been evaluated as probes for these imaging modalities (Table 1).

\subsubsection{Single photon emission computed tomography (SPECT)}

The first trial to test aptamers in molecular imaging was performed in 1997 by Charlton et al. [27]. This study used a DNA aptamer (called NX21909) that binds to neutrophil elastase, which is a serine protease secreted by neutrophils during inflammation. This aptamer was radiolabeled with ${ }^{99 \mathrm{~m}} \mathrm{Tc}$ and intravenously injected into rats with induced inflammation in a forelimb. The biodistribution of the 
aptamer was measured by scintigraphy and compared to that of a reference $\operatorname{IgG}$ used clinically to image inflammation and a negative control oligonucleotide. Every compound exhibited a higher signal in the inflamed forelimb than the contro-lateral healthy forelimb. In addition, a higher signal was measured with the aptamer than the control oligonucleotide in the inflamed forelimb. Finally, the control oligonucleotide and the aptamer showed faster clearance than the reference $\operatorname{IgG}$. This led the aptamer to reach a signal-to-noise ratio of $4.3 \pm 0.6$ just two hours post-injection, whereas the best ratio obtained by the antibody was only $3.1 \pm 0.1$ and occurred three hours post-injection.

Hicke et al. further evaluated an aptamer using SPECT for cancer imaging in 2006 [28]. This aptamer, called TTA1, was selected against tenascin $\mathrm{C}(\mathrm{TN}-\mathrm{C})$, an extracellular matrix protein overexpressed in many tumors. This aptamer was a 2'fluoro-pyrimidine RNA oligomer. Accordingly, every pyrimidine of this aptamer contained a fluor group in the 2' position of the ribose; this chemical modification is known to provide greater resistance against degradation by nucleases. In contrast, the previously described anti-elastase aptamer used natural DNA chemistry, which is expected to be rapidly degraded by nucleases in vivo. The biodistribution of the TTA1 aptamer was measured by SPECT imaging after intravenous injection in mice bearing subcutaneous tumor xenografts from human U251 glioblastoma cells. A maximum uptake of $6 \%$ of injected dose/g (ID/g) was achieved in the tumor 10 minutes after intravenous injection of $\left[{ }^{99 \mathrm{~m}} \mathrm{Tc}\right]$-radiolabeled TTA1. The aptamer in the tumor then continuously decreased, but $1.9 \% \mathrm{ID} / \mathrm{g}$ was still present three hours post-injection. A negative control aptamer showed initial tumor absorption that was half of that of TTA1 ( $3 \%$ ID/g) at 10 minutes but it was rapidly cleared from the tumor, with only $0.04 \%$ ID/g still present three hours post-injection. This aptamer demonstrated rapid blood clearance and reached a tumor-to-blood ratio of approximately 50 just three hours after injection. Such rapid clearance can be explained by rapid renal excretion, as well as nuclease degradation. Indeed, the aptamer detected in the blood three hours after injection was completely degraded, whereas the radioactivity present in the tumor at that time corresponded mostly to non-degraded aptamer. The authors suggested that the aptamer could have been partially protected against nucleases when bound to its target. This study also examined the importance of the radiometal chelator and the injected dose in the biodistribution of the probe. Three different chelators were compared for radiolabeling of the aptamer. The clearance of the aptamer was markedly different from the three chelators, although they had less of an effect on tumor targeting. Finally, increasing the injected dose was shown to correlate with increased circulation time of the aptamer in blood and higher absorption by the tumor, suggesting that the clearance of this aptamer should be saturable.

Several other aptamers have been evaluated by SPECT imaging for tumor targeting (see Table 1). They were mostly selected against proteins that are overexpressed at the surface of cancer cells. 
These studies confirmed that aptamers are rapidly cleared from the bloodstream and eliminated either by hepatobilary and/or urinary excretion. Two DNA aptamers, selected against Mucin 1 (MUC1) [29] and nucleolin [30], demonstrated higher uptake in the tumors than muscle, suggesting that they can bind to their targets in vivo. These aptamers were, however, evaluated without comparing them to a negative oligonucleotide control. Thus, its impossible to know whether tumor uptake was linked to their interaction with their target or to higher vascularization of the tumor. For example, similar tumor uptake was observed with a 2' F-Py RNA aptamer selected against human epidermal growth factor receptor 2 (HER2), but comparison of the aptamer with that of a control sequence showed the two to have the same tumor uptake [31]. This demonstrates the importance of including this control in such studies to determine whether tumor uptake of an aptamer is non-specific or results from an interaction with its target.

Accordingly, two aptamers demonstrated significantly higher tumor uptake than a control oligonucleotide: a DNA aptamer selected against epidermal growth factor receptor variant III (EGFRvIII) [32] and a fully modified aptamer (2'Ome-Purine/2'F-Py) that binds to matrix metalloprotease-9 (MMP9) [33, 34]. The latter was radiolabeled either with ${ }^{99 \mathrm{~m}}$ Tc-MAG or ${ }^{111} \mathrm{In}$ DOTA [34]. Significant tumor targeting was observed for both labels, but high hepatobilary excretion was observed for the ${ }^{99 \mathrm{~m}}$ Tc-MAG-labeled aptamer, whereas the ${ }^{111}$ In-DOTA-labeled aptamer was mostly eliminated by the urinary pathway. This result confirmed that labeling can strongly influence the biodistribution of aptamers.

Recently, three studies published by the group of Antero Silva Ribeiro de Andrade investigated the use of aptamers as probes for the detection of bacterial infections by SPECT imaging [35-37]. These aptamers were selected against various structural cell-wall components of bacteria. Scintigraphy of Staphylococus aureus infected mice using ${ }^{99 \mathrm{~m}}$ Tc-radiolabeled aptamers demonstrated that the bacterial infection could be located, with target to non-target ratios that were statistically higher than those achieved for a control sequence.

\subsubsection{Positron emission tomography (PET)}

Four studies have evaluated the use of aptamers as probes for PET imaging in several cancer models (Table 1). Two used a DNA aptamer (AS1411) that binds to nucleolin. This protein, overexpressed on the surface of many cancerous cells, can internalize when it binds the aptamer. In 2014, Li et al. conjugated the extremity of the AS1411 aptamer with four different chelators, allowing radiolabeling with ${ }^{64} \mathrm{Cu}$ [38]. They demonstrated that these chelators can substantially alter internalization of the aptamer in vitro. They also demonstrated that the biodistribution and tumor targeting of this aptamer changes, depending on the chelator used, similar to the SPECT imaging 
studies. The same AS1411 aptamer was also evaluated by Park et al. using a control sequence [39]. In this study, the aptamer was elongated by a sequence of 15 nucleotides that was then hybridized to an antisense oligonucleotide previously radiolabeled with ${ }^{18} \mathrm{~F}$ by click chemistry. This clever methodology makes it possible to easily radiolabel several aptamers at the same time using the ${ }^{18} \mathrm{~F}$ radiolabeled antisense oligonucleotide. The efficacy and specificity of tumor targeting of this aptamer was evaluated by micro-PET in mice carrying tumor xenografts. High absorption was observed in the kidneys, intestines, and liver for both the aptamer and the control sequence. However, the aptamer showed slightly higher tumor targeting than the control sequence. They carried out a blocking experiment to confirm the specificity of tumor targeting by co-injecting a 100-fold molecular excess of unlabeled AS1411 aptamers. Tumor uptake was reduced by a factor of two, suggesting that part of the tumor targeting was possibly specific.

In 2015, Jacobson et al. evaluated two other aptamers as probes for PET imaging [40]. First, they evaluated an aptamer against tenascin-C. They used a DNA aptamer that is completely different from the 2'-fluoro-Py RNA aptamer previously studied by SPECT imaging (see above). It was radiolabeled with either ${ }^{64} \mathrm{Cu}$ or ${ }^{18} \mathrm{~F}$ before being evaluated by PET imaging in mice bearing a subcutaneous tumor xenograft. Although this aptamer was described to have poor binding at $37^{\circ} \mathrm{C}(\mathrm{Kd}$ in the $\mu \mathrm{M}$ range) [41], it demonstrated higher tumor uptake than a scrambled control sequence for both labeling strategies. However, the 2'fluoro-Py-labelled aptamer, previously evaluated by SPECT, showed 10-fold higher uptake by the tumor one hour after injection (3 versus $0.3 \% \mathrm{ID} / \mathrm{g}$ ) and also demonstrated a 10-fold higher tumor/blood ratio three hours after injection (50 versus 4 ).

Then, the same group studied a DNA aptamer selected against protein tyrosine kinase-7 (PTK7), a member of the receptor tyrosine kinase superfamily that is highly expressed in various human malignancies [42]. The aptamer was evaluated in mice bearing subcutaneous tumors from the HCT116 and U87MG cell lines. PET studies showed specific accumulation of the ${ }^{18}$ F-radiolabeled aptamer in HCT116 tumors, which express high levels of PTK7, and lower uptake by U87MG tumors, which express lower levels of PTK7. Uptake of the aptamer was higher than that of the control sequence in both models. The specificity was also confirmed by an $80 \%$ inhibition of uptake when the radioactive aptamer was co-injected with a large excess of unlabeled aptamer. Furthermore, the aptamer demonstrated higher uptake by liver metastases from HCT116 cells than by the subcutaneous tumor. Finally, they found that increasing the injected dose increased tumor targeting of the aptamer, as previously observed.

\subsection{In vivo fluorescence imaging with aptamers}


In vivo fluorescence imaging is similar to fluorescence microscopy but at a macroscopic level. This type of imaging has become widespread in laboratories. However, unlike other molecular imaging techniques, the use of fluorescence imaging is technically more difficult because biological tissues absorb and disperse photons and can also generate strong autofluorescence, obscuring the collection and quantification of signals. Nevertheless, significant progress has been made in the mathematical models that describe the propagation of photons in tissues. Moreover, the illumination and detection instruments available have improved and this type of imaging is now widely used for small rodent imaging. In addition, it shows promise for several applications in humans, especially for image-assisted surgery.

Many fluorescent probes have been designed using aptamers (Table 1). The simplest way is to graft the aptamer with a small organic dye. Generally, fluorophores emitting in the near infrared are preferred because biological tissues absorb less photons and have less autofluorescence at these wavelengths. The fluorescent aptamer is then intravenously injected into living subjects and fluorescence imaging is performed at various times. Usually, "planar imaging" is used for qualitative or semi-quantitative 2D imaging. This technique uses wide-field illumination for the excitation of a fluorophore inside animals and a highly sensitive camera to record the fluorescence signals. Planar imaging is strongly affected by light scattering. Thus, it is mainly used for imaging surface tissues, such as subcutaneous tumor xenografts, comparing the fluorescence signal inside the tissue of interest with the background signal originating from a reference area [43]. This approach has been used with many aptamers that bind to proteins overexpressed at the surface of cancer cells (Table 1). They have generally demonstrated higher fluorescence signals of the tumor than that of the background from a few minutes after injection up to several hours [44-48].

In vivo visualization and quantification of the fluorescence signal have been improved by the development of fluorescence diffuse optical tomography (fDOT). This imaging technique, also called fluorescence molecular tomography (FMT), requires instruments that have a transillumination mode for excitation, generally by lasers. In contrast to planar images, fDOT can reconstruct the original fluorescence emission signal in three dimensions using algorithms. We previously compared fDOT and PET to monitor the biodistribution of oligonucleotides inside mice [49]. Although fDOT was less sensitive, it was able to measure similar concentrations of oligonucleotides ranging from $3 \mathrm{nM}$ to 1 $\mu \mathrm{M}$. We were able to more precisely quantify the tumor targeting of an anti-annexin A2 aptamer using this technique [46]. Three hours after injection, the quantity of this aptamer in the tumor was approximately 14 times higher than that of a control sequence. fDOT was also used by Mi et al. to quantify the tumor targeting of an aptamer that binds to DHX9, an RNA helicase protein [50]. In this study, the aptamer was evaluated in an animal model of hepatic colorectal cancer metastases. In 
contrast to a subcutaneous xenograft, it was difficult to evaluate the size of such tumors, that can vary highly between animals. Nevertheless, one of the major advantages of fluorescence imaging is that it can measure several fluorophores at the same time. The authors used this property to normalize the tumor targeting of their aptamer by another fluorescent probe that targets the tumor.

A major drawback of molecular imaging is the difficulty to discriminate the specific binding of a probe from its non-specific distribution. However, unlike other types of imaging, in which the probes continuously produce a signal over time, it is possible to modulate fluorescence emission of a fluorophore according to its environment. This property has been used to develop activatable probes, also called "smart fluorescent probes", that can produce a fluorescent signal only when they are bound to their target. Hence, Shi et al. developed an activatable aptamer probe (AAP) using a DNA aptamer targeting PTK7 [51]. They produced this AAP by elongating the aptamer with a poly-thymidine sequence and partially hybridizing it to a short DNA sequence containing a fluorophore and a quencher attached at each extremity. In the absence of the target, the construct forms a hairpin structure that brings the fluorophore close to the quencher, resulting in quenching of the fluorescence. However, when the aptamer is bound to the membrane receptors of cancer cells, its conformation changes, resulting in an activated fluorescence signal. This construct was evaluated in mice bearing subcutaneous tumors. The AAP displayed substantially enhanced contrast relative to classical labeling, in which the aptamer is continuously fluorescent. Furthermore, such enhanced contrast was achieved within 15 minutes after intravenous injection, whereas the always-on probe required more time to achieve maximal contrast because of the need to wait for clearance of the unbound probe.

Li et al. designed another activatable fluorescent probe with a DNA aptamer that binds to cellsurface MUC1, which is overexpressed in many malignant tumors [52]. In this case, the Cy3-labeled aptamer was $\pi-\pi$ stacked on the surface of oxidized mesoporous carbon nanospheres, resulting in quenched fluorescence. After exposure to MUC1, the aptamer is gradually released from the oxidized mesoporous carbon nanosphere due to a much stronger interaction with the protein, resulting in fluorescence recovery. Although this construct led to promising in vitro results, it needs to be optimized for in vivo use. Indeed, a targeted fluorescenct signal in the tumor was achieved by intratumoral injection, but not intravenous injection.

Recently, two aptamers were tested by fluorescence imaging in animal models of Alzheimer's disease [53, 54]. One aptamer, called $3-55$, binds to amyloid fibrils found in Alzheimer's disease but not the A-\$40 monomer. Farrar et al. fluorescently labeled this aptamer and used it to visualize amyloid plaques in APP/PS1 transgenic mice in vivo using two-photon imaging [53].

\subsection{Magnetic resonance imaging (MRI) with aptamers}


MRI uses strong magnetic fields, electric field gradients, and radio waves to generate images. MRI is known to have very high spatial resolution, but is relatively insensitive. Contrast agents have been developed to improve sensitivity, including T1-positive paramagnetic agents and T2-negative superparamagnetic nanoparticles. A few studies have been conducted to specifically address the use of aptamers to improve the imaging of tumors using contrast agents [55-58]. The aptamers studied bind to proteins that are expressed on the surface of proliferating endothelial cells during angiogenesis, such as nucleolin, ævß3 integrin, vascular endothelial growth factor, and vascular endothelial growth factor receptor type 2 (VEGFR2). The fact that MRI contrast agents are sometimes large (up to several hundred $\mathrm{nm}$ in diameter), may explain why trials mostly targeted biomarkers that do not require extravasation from the bloodstream to be reached. These studies all demonstrated improved MRI contrast in tumors relative to nanoparticles without aptamers. However, no one compared signals using the same nanoparticle conjugated with a negative-control oligonucleotide, although conjugation of a negatively charged oligonucleotide can substantially affect the biodistribution of a nanoparticle. Thus, as already mentioned, it is difficult to know whether the signal is due to the interaction of the aptamer with its target or to higher non-specific uptake of the nanoparticle by the tumor.

\subsection{Ultrasound imaging}

Ultrasound imaging, also called echography, is a non-invasive method widely used in clinical settings for real-time anatomical and functional imaging. It uses a probe to send ultrasound pulses and record the sound echoes off the tissues. These echoes can be used to reconstruct an image, as different tissues reflect varying degrees of sound. The most common contrast agents used in molecular ultrasound are micron-sized, gas-containing microbubbles. Nakatsuka et al. functionalized such

microbubbles with an aptamer that binds to thrombin [59]. Thrombin acts in the blood coagulation pathway to convert soluble fibrinogen into insoluble strands of fibrin, as well as catalyze other coagulation-related reactions. They observed a five-fold increase in acoustic activity in the clot region after injection of the microbubbles in a static thrombosis model in a rabbit. However, they did not compare their results with those using the same microbubbles conjugated with a control sequence.

\section{Multimodal imaging with aptamers}

Each imaging modality has advantages and disadvantages. Thus, there are an increasing number of hybrid instruments that offer the possibility of combining several imaging modalities. Similarly, multimodal imaging probes based on nanoparticles are being developed for detection by different imaging modalities. Several such probes have used aptamers as specific targeting agents (Table 2). For example, Hwang et al. used a cobalt-ferrite nanoparticle surrounded by fluorescent 
rhodamine in a silica shell matrix [60]. This nano-object, already detectable by MRI and fluorescence imaging, was conjugated with an anti-nucleolin aptamer before being further radiolabeled with ${ }^{67} \mathrm{Ga}$ for detection by SPECT imaging. They then evaluated the biodistribution of this multimodal-imaging probe in mice bearing subcutaneous tumor xenografts. As a negative control, they used the same construct in which they replaced the aptamer by a mutant aptamer in which several guanines were substituted with cytosines. Scintigraphy revealed higher tumor absorption of the nanoparticles conjugated to the aptamer than for those conjugated to the control sequence 24 hours after intravenous injection. T2-weighted MR images of the same mice detected the presence of nanoparticles in tumors only when conjugated with the aptamer. Finally, higher tumor uptake of the nanoparticles conjugated to the aptamer was further confirmed by ex vivo fluorescence imaging of extracted organs. As expected, SPECT imaging was more sensitive and provided better quantification, whereas MRI provided better resolution. Apart from preclinical research, the use of this type of probe in humans is debatable. Indeed, the design of multimodal imaging probes is necessarily more complicated and costly. Furthermore, combining SPECT and MRI to detect the presence of a tumor does not provide any additional information over the use of either of these modalities alone. A more optimal approach would be to use a dual-modality probe that can be used during an initial whole-body examination to identify the location of a tumor and then assist in locating it during tumor resection surgery. Thus, most existing multimodal imaging probes combine fluorescence, which can be detected during surgery, with a whole-body imaging modality, such as PET, SPECT, MRI, or X-ray CT. For example, Li et al. conjugated the anti-nucleolin aptamer to fluorescent gold nanoparticles, which can be detected both by X-ray CT and fluorescence imaging [61]. This dual-modal imaging probe was detected in tumors by $\mathrm{CT}$ imaging 30 minutes after intravenous injection in mice bearing subcutaneous tumor xenografts. Furthermore, the tumor can be easily visualized during surgery under ultraviolet light, due to an orange-red fluorescent signal emitted from the nanoparticles. Such tumor targeting was not achieved by the same nanoparticle without an aptamer; however, further experiments should be performed with a control oligonucleotide to determine whether the enhanced contrast is due to interaction of the aptamer with its target.

\section{Use of aptamers for theranostics}

The term "theranostics" is used to define the research that is carried out to combine diagnostic and therapeutic capabilities into a single agent and develop more specific individualized therapies. This is because many existing therapies are effective only for a limited number of patient subpopulations and at selective stages of the disease. Thus, a nano-object that can co-deliver a therapy and an imaging agent could allow imaging to be used during treatment to determine whether the treatment is reaching 
the pathological area. It would therefore be possible to provide more specific therapeutic protocols to individuals to likely provide a better prognosis. Another advantage offered by theranostics is the simultaneous improvement of treatment efficiency and reduction in side effects for patients by specific targeting with the aptamer. Indeed, the treatment should be specifically addressed to the disease area instead of being spread throughout the body, with the result that healthy tissues receive a lower dose. Several theranostic strategies using aptamers have been evaluated in vivo in cancer models (Table 3). For all these strategies, it is necessary to use a conjugation method to attach the aptamer at the nanoparticle surface and to verify that the aptamer conserve its binding capacity. In addition, several conjugation methods could be evaluated to define the best spacer between aptamers and nanoparticles, as well as to define the best number of aptamers to conjugate per nanoparticle [62].

\subsection{Delivery of anthracycline drugs and imaging}

Most theranostic studies with aptamers concern the co-delivery of imaging agents and anthracycline drugs (predominantly doxorubicin). These drugs interact with DNA by intercalation, inhibiting replication, and deregulating gene expression. The first way to deliver the drugs was to load it in various types of nanoparticles that were further labeled for detection by imaging and conjugated to aptamers. This approach was used by Pascual et al. who loaded doxorubicin in mesoporous silica nanoparticles that were further conjugated with an anti-MUC1 aptamer and radiolabeled by ${ }^{99} \mathrm{Tc}$ [63]. Specific targeting and drug delivery of the nanoparticle was demonstrated in vitro on cells and scintigraphy showed that the nanoparticles can target a subcutaneous tumor in vivo. However, the in vivo biodistribution was not compared to that of the same nanoparticle conjugated with a control sequence. A similar strategy was used by Mosafer et al. who entrapped superparamagnetic iron oxide nanoparticles (SPIONs) and doxorubicin in poly(lactic-co-glycolic acid) (PLGA)-based nanoparticles that were further conjugated to the anti-nucleolin aptamer [64, 65]. Higher tumor targeting was observed by MRI for the nanoparticles conjugated with the aptamer than for unconjugated nanoparticles. Furthermore, the nanoparticles conjugated with the aptamer provided significantly higher inhibition of tumor growth and prolonged survival of mice bearing C26 colon carcinoma xenografts.

Another method to deliver drugs is to covalently attach them to the surface of nanoparticles. This strategy was used by Salva et al. who conjugated an anti-MUC1 aptamer to Quantum Dots and further attached doxorubicin to their surface via a $\mathrm{pH}$-sensitive hydrazone bond [66]. These bonds are stable in general circulation at a neutral and slightly basic $\mathrm{pH}$ but undergo rapid hydrolysis in the acidic environment of endosomes. Accordingly, it should release the drug specifically in cancer cells that express nucleolin because they should internalize the nanoparticles via the aptamer. In vivo 
fluorescence imaging demonstrated that the Quantum Dots accumulated more in subcutaneous xenografts when they were conjugated to the aptamer. Furthermore, the therapeutic efficiency of theses nanoparticles was slightly improved by the aptamer in vitro, but were not evaluated in vivo.

Doxorubicin was also conjugated to the surface of luminescent gold nanoclusters by Chen et al. [67]. They further conjugated this nanoparticle with two targeting moieties: the anti-nucleolin aptamer and a cyclic Arg-Gly-Asp (cRGD) peptide, which binds to $\alpha_{v} \beta_{3}$ and $\alpha_{v} \beta_{5}$ integrins, which are upregulated in tumor endothelial cells. In vivo fluorescence imaging demonstrated significantly greater tumor targeting when the nanoparticles were conjugated with the dual targeting agents than when they were unconjugated, and slightly greater tumor targeting than nanoparticles conjugated with only cRGD. Intravenous injection of the nanoparticles conjugated with the dual targeting agents every 48 hours induced greater inhibition of tumor growth than unconjugated nanoparticles and free doxorubicin.

Finally, the property of anthracyclines to intercalate into DNA has been used to load nanoparticles. Hence, $\mathrm{Yu}$ et al. used an aptamer that binds to the prostate-specific membrane antigen (PSMA), which is over expressed on some prostate tumors [68]. They elongated the sequence of the aptamer by an additional $(\mathrm{CGA})_{7}$ repeat that was hybridized to a sequence previously conjugated to the surface of SPIONs. This double strand pairing was not only used to conjugate the aptamer but also for the loading of doxorubicin, which is known to predominantly bind to consecutive CG base pair regions. The biodistribution of this complex was then evaluated by MRI in nude mice bearing subcutaneous tumors. From one hour after injection, the nanoparticles conjugated with the aptamer demonstrated ten-fold higher tumor targeting than those conjugated with a scrambled control sequence. They then evaluated the in vivo therapeutic efficiency by injecting the nanoparticles every five days for 15 days. The mean fold increase in tumor volume (from day 0 to day 25) in mice was significantly less when the nanoparticles were conjugated to the aptamer than when a scrambled sequence was used $(3.4 \pm 0.6$-fold $v s 5.2 \pm 0.6$-fold $)$ or free doxorubicin $(5.0 \pm 0.4$-fold $)$. A similar loading strategy was also used by Jalalian et al. who loaded a MUC1 aptamer, that was conjugated to SPIONs, with epirubicin [69]. MRI demonstrated higher tumor targeting of the nanoparticles conjugated with the aptamer than unconjugated nanoparticles, similarly to the previous study. Furthermore, they also demonstrated greater inhibition of tumor growth.

The property of doxorubicin to intercalate into DNA was also used by Lei et al. to develop a smart split aptamer-based activatable theranostic probe [70]. They used the Sgc8c aptamer that interacts with a cancer-associated membrane PTK-7. The Sgc8c aptamer was split into two parts and linked via a short DNA linker labeled with a fluorophore. The central part of this linker was then hybridized with a complementary short DNA strand to which was attached a quencher that abolishes 
the fluorescence emission of the fluorophore. It was also loaded with doxorubicin, as the double helix region linker/short DNA strand was GC rich. The construct was designed to disassemble when the structure of the aptamer changes during its interaction with PTK-7, releasing the doxorubicin and activating the emission of a fluorescent signal. Both therapy and imaging were validated in vitro. They also evaluated the construct in vivo by fluorescence imaging in nude mice bearing subcutaneous tumors. The tumor showed high fluorescence just 15 minutes post-injection, but the therapeutic efficiency was not evaluated.

\subsection{Delivery of taxane drugs and imaging}

Several theranostic studies have been performed with aptamers for the co-delivery of imaging agents and taxane drugs, such as paclitaxel (Taxol) and docetaxel (Taxotere), which are widely used as chemotherapy agents. The principal mechanism of action of these drugs is to disrupt the microtubule functions that are essential for cell division. In contrast to anthracyclines, which are hydrophilic, taxanes are hydrophobic. Thus, the theranostics nanosystems developed for those drugs are different.

Tao et al. used a nano-precipitation method with an acetone/water system to prepare nanoparticles of cholic acid functionalized with a star-shaped block copolymer consisting of PLGA and vitamin E TPGS loaded with docetaxel and a near infrared fluorescent dye [71]. The surface of these nanoparticles was further conjugated with the anti-nucleolin aptamer. The resulting nanoparticles were evaluated in two mouse models in which the mice received subcutaneous tumor xenografts from human breast cancer cell lines. The nanoparticles conjugated with the aptamer demonstrated four-fold higher tumor targeting 24 hours after intravenous injection than unconjugated nanoparticles or free dye by fluorescence imaging. Furthermore, they also demonstrated greater inhibition of tumor growth.

The anti-nucleolin aptamer was also used by Luo et al. to target poly (L-c-glutamyl-glutamine) nanoconjugates loaded with paclitaxel and a fluorescent dye [72]. In contrast to most of the studies described previously, these nanoparticles were not evaluated in subcutaneous models, but in nude mice that develop orthotopic brain tumors from intracranial injection of human U87 MG glioblastoma cells. Fluorescence imaging revealed two-fold higher tumor targeting by the nanoparticles conjugated with the aptamer than unconjugated nanoparticles 24 hours after intravenous injection. Furthermore, when the nanoparticles were injected every three days, the median survival of mice that received the nanoparticles (52 days) was significantly longer than those treated with unconjugated nanoparticles (47 days), free Taxol (40 days), or a control saline solution (36 days). Similar results were obtained by Gao et al. in another orthotopic brain tumor model. They also used the anti-nucleolin aptamer attached to the surface of poly(ethylene glycol)-poly(e-caprolactone) nanoparticles loaded with docetaxel and fluorescence dye [73]. The surface of the nanoparticle was also conjugated with a peptide previously 
screened by phage display to cross the blood brain barrier (BBB). Fluorescence imaging demonstrated that this peptide enhanced brain uptake of the nanoparticles and that addition of the aptamer increased uptake by the tumor. Furthermore, injection of the dual-targeting nanoparticle every three days, for a total of three injections, significantly improved the median survival of mice over those that received the nanoparticles with only one targeting agent. The same nanoparticle achieved the same result using another aptamer selected to recognize glioblastoma cancer cells, but without the need of the peptide to cross the BBB [74]. This may be explained by the fact that the BBB is perhaps disrupted during growth of the brain tumor.

\subsection{Delivery of nucleic acid drugs and imaging}

Two studies have described the use of aptamers for the codelivery of nucleic acid drugs and an imaging agent. Kim et al. developed a fluorescent-activatable probe that can detect the expression of a miRNA and simultaneously inhibit its function [75]. This probe was constructed by conjugating the anti-nucleolin aptamer and a fluorescent molecular beacon to the surface of magnetic-fluorescent nanoparticles. The molecular beacon was designed to provide fluorescence emission upon hybridization with the micro-RNA (miRNA) 221. This interaction can also inhibit the function of miRNA 221, which promotes cancer by an antisense-like effect. C6 cells were first transfected by the probe before being subcutaneously implanted into nude mice. In vivo fluorescence imaging revealed that the miRNA could be detected in cancer cells for up to four days. The probe was also injected directly into already implanted subcutaneous tumors. It significantly decreased tumor growth relative to injections with PBS.

Recently, an anti-EGFR aptamer was used to target liposomes containing Quantum dots for fluorescent imaging and siRNA molecules for therapy. At four hours post-injection, nanoparticles conjugated with the aptamer demonstrated two-fold higher tumor targeting than unconjugated nanoparticles. However, the fluorescence was cleared from the tumor by 24 hours and the therapeutic efficiency was not evaluated.

\subsection{Photodynamic therapy (PDT) and imaging}

Photodynamic therapy (PDT) uses a photosensitizing chemical substance that produces molecular oxygen when excited by light, causing cell death. Tian at al. in 2014 attempted to perform photodynamic therapy using nanomicelles encapsulating a $\mathrm{pH}$ activatable fluorescent probe and a near-infrared photosensitizer, called R16FP, as a theranostic [76]. The nanomicelles were targeted by an aptamer previously selected against MDA-MB-231 human breast cancer cells. The goal was to use 
the fluorescent dye to detect when nanomicelles reached the lysosomes of cancer cells to determine when to carry out NIR irradiation of the tumor region to induce the PDT. Upon irradiation, R16FP mediates the generation of reactive oxygen species (ROS) and can thus cause lysosomal destruction and subsequent cell death. In vivo fluorescence images of subcutaneous MDA-MB- 231 tumor-bearing mice were acquired at various times post-i.v. injection. The tumor/background fluorescence signal ratio gradually increased and reached a maximum 24 hours after injection. Furthermore, tumor targeting was higher for nanoparticles conjugated to aptamers than those that were unconjugated or conjugated to a scrambled control sequence. PDT was evaluated by NIR irradiation of the tumor 24 hours post-injection. Treatment resulted in the inhibition of tumor growth and the tumor tissues showed noticeable necrosis. Furthermore, histological analysis of several organs from the PDT-treated mice showed no signs of injury, suggesting insignificant toxic side effects.

\subsection{Photothermal therapy (PTT) and imaging}

Photothermal therapy (PTT) uses electromagnetic radiation (mostly infrared wavelengths) to bring a sensitizer to an excited state, at which point it then releases vibrational energy (heat) that can kill the targeted cells. Two studies have described the use of fluorescently-labeled nanoparticles targeted by aptamers for guided PTT. In 2016, Ye et al. used $\mathrm{Cu}-\mathrm{Au}$ alloy nanostructures as sensitizers addressed by the Sgc8c aptamer, which targets PTK7 [77]. This system was labeled with Cy5 for fluorescent imaging. In vivo fluorescence imaging revealed higher tumor targeting by the targeting nanoparticles than those conjugated with a negative control scrambled sequence two hours after intravenous injection into CCRF-CEM tumor bearing mice. PTT was evaluated in mice that received an intratumoral injection of the nanoparticles and subsequently exposed to $980 \mathrm{~nm}$ irradiation for five minutes. They observed tumor necrosis and even complete disappearance of the tumor four days after PTT treatment. The second study, by Shi et al. in 2014, used Au(core)/Ag-Au(shell) nanoparticles as sensitizers [78]. These nanoparticles were conjugated to an activatable aptamer probe (AAP) with quenched fluorescence in the free state. As previously described, such probes can emit fluorescence when bound to their target. Under the guidance of this fluorescent imaging signal, PTT was performed in nude mice bearing tumor xenografts by exposing the tumor to a $980 \mathrm{~nm}$ laser for five minutes. The tumor gradually became necrotic and tumor growth was inhibited at the necrotic site.

Two other studies used the PTT strategy, not only to kill cancer cells but also to control delivery of the drugs to inside the tumors. The first was conducted in 2006 by Chuang et al. [79]. Doxorubicin was encapsulated, with ammonium bicarbonate and gold nanocages, into liposomes conjugated to MUC1 aptamers. Exposure of the gold nanocages to NIR light can produce localized heat and the heating of ammonium bicarbonate generates $\mathrm{CO} 2$ bubbles. This can disrupt liposomes and 
locally liberate doxorubicin. An activatable aptamer probe was used to monitor the interaction of aptamer with its target. The signal was maximal at the tumor site 30 minutes post-injection and disappeared by one hour, suggesting that the nanoparticles were rapidly internalized. Thus, mice were injected every four days with the liposome and tumors were subjected to PTT one hour after injection. The size of the tumor decreased over time, whereas it increased in untreated mice or mice treated with free doxorubicin. Furthermore, the size of the tumor decreased less using the same theranostic system without NIR irradiation.

In 2017, Zhao et al. used the same theranostic strategy [80]. However, the drug encapsulated in the liposomes was docetaxel and the system was targeted by two aptamers. The liposomes were coated with gold nanoshells, which served as sensitizers. Inhibition of tumor growth in mice bearing S180 cell tumors was also observed relative to several control groups, including mice injected with non-targeted liposomes. Ultrasound imaging was also performed: such liposomes provided better tumor resolution and allowed longer observation than Sonovue, a commonly used ultrasound contrast agent.

\subsection{Drug delivery and multimodal imaging}

In 2015, Roy et al. performed one of the first trials of combination drug delivery and multimodal imaging [81]. They coupled NIR, MRI, and CT imaging with the delivery of bovine lactoferrin, an emerging anticancer drug [82]. $\mathrm{Fe}_{3} \mathrm{O}_{4}$ and bovine lactoferrin were encapsulated in alginate-enclosed, chitosan-conjugated, calcium phosphate nanocarriers. The resulting nanoparticles were targeted with locked nucleic acid (LNA) aptamers against epithelial cell adhesion molecules and nucleolin. In vivo therapeutic efficiency of these nanoparticles was evaluated in subcutaneous tumor bearing mice that were orally fed the nanoparticles for 45 days. Complete regression of the tumors was observed for $70 \%$ of the mice fed the nanoparticles. Most importantly, mice injected with aptamertargeted nanoparticles showed three-fold lower tumor recurrence after 30 days than those injected with non-targeted nanoparticles. Three imaging techniques were used to measure specific internalization of the targeted nanoparticles into the tumors. For fluorescent imaging, VivoTag 680 XL was encapsulated into the nanoparticles and the nanoparticles fed to the mice for $48 \mathrm{~h}$. The fluorescence intensity of the tumor was higher for mice fed the aptamer-targeted nanoparticles than those fed the non-targeted nanoparticles. MRI and X-ray CT imaging confirmed higher tumor targeting. 


\section{Conclusions \& perspectives}

Although aptamers represent a promising class of ligands, their use as molecular imaging probes is relatively new. Nonetheless, the number of in vivo preclinical studies using aptamers has increased significantly in recent years (Figure 4). To date, the reported applications of aptamers for molecular imaging and theranostics have been almost exclusively focused on cancer (Figure 4C) and half have focused on four targets (Figure 4D). This is partially because most aptamers are currently selected against cancer biomarkers. However, the scope of application should extend to other diseases, such as neurodegenerative and infectious diseases, as the number of aptamers identified each year increases [83, 84].

A number of issues are still largely unresolved. For example, the degradation of these imaging probes in vivo requires further study. This information is missing from almost all published studies while it is mandatory to better interpret the imaging results. For example, there is an increasing use of natural DNA aptamers whereas it is known that DNA degrades rapidly in plasma, which raises questions about the validity of results obtained several hours after injection. Another issue is the potential toxicity of aptamers that is almost never studied. Aptamers are often considered to have low immunogenicity and low toxicity. However oligonucleotides could potentially be recognized by the innate immune system via germline-encoded-pattern-recognition receptors (PRRs) like Toll-like receptors (TLRs) [85]. Accordingly, aptamers immunogenicity should be more studied before further development. Moreover, their fast excretion through renal clearance could also limit the use of aptamers. One of the solutions is to conjugate the aptamer with high molecular weight molecules that can reduce the renal filtration. For instance, the conjugation of the aptamer with polyethylene glycol (PEG) has been widely used [11]. However, such conjugation could affect the affinity of aptamers. Additionally, too few studies have compared the biodistribution of the imaging probe with that of one comprised of a random sequence of the same size and chemistry. Without this important negative control, it is impossible to determine whether the tumor targeting observed in many studies is due to the interaction of the aptamer with its target or improved passive tumor targeting by the so call "enhanced permeability effect". Indeed, new blood vessels formed during tumor growth are usually disorganized and contain wide pores. In addition, tumors exhibit poor lymphatic drainage. Both of these phenomena can promote the diffusion and trapping of molecular imaging probes within the tumor interstitium, without the need to bind to a specific molecular target [86].

Several studies have also demonstrated that labeling can affect the biodistribution of aptamers and also affect their binding. However, very few studies have measured the dissociation constant (Kd) of their molecular probes, although this information is crucial for comparing studies, especially those that are performed with the same aptamer, but different labeling. Finally, most studies have been 
conducted in nude mice bearing tumor xenografts. However, many studies have demonstrated the limitations of such models, which have little resemblance to real cancer. It would thus be very informative to test the biodistribution of aptamer-based imaging probes on other cancer models, such as transgenic mice that develop metastases.

In conclusion, the use of aptamers to develop imaging probes or theranostics agents is still in its infancy, but the successful preclinical results already achieved in a short time are impressive. Several studies have already demonstrated significant proof of concept, which may lead to further progress towards clinical trials in the future. 


\section{Acknowledgments}

Our aptamer studies are supported by grants from Investissement d'Avenir ANR-11-INBS-0011 NeurATRIS: A Translational Research Infrastructure for Biotherapies in Neurosciences. ABM was supported by a PhD fellowship (CEA-irtélis).

\section{Conflict of Interest}

The authors declare no conflict of interest. 
Figure 1. Scheme of aptamer-based molecular imaging probes. A) An aptamer is conjugated by a linker to a molecule (label) that allows detection by a molecular imaging instrument. Such probes are generally used for nuclear or fluorescence imaging aided by radioisotopes or fluorescent dyes, respectively. B) For MRI and CT imaging techniques, aptamers are linked to contrast agents based on nanoparticles. These nanoparticles can be simultaneously labeled with several labels to build multimodal imaging probes. They can also be used to co-deliver drugs for theranostic applications.

Figure 2. Principle of molecular imaging. At $t_{0}$, the contrast agent (in green) is injected inside the subject. Then, it spreads over the whole body $\left(t_{1}\right)$ and is finally eliminated via excretory organs, except in the area of interest where the probe interacts with the target $\left(t_{2}\right)$.

Figure 3. General principle of aptamer selection by systematic evolution of ligands by exponential enrichment (SELEX). A random oligonucleotide library is incubated with a target (1). Sequences that bind to the target are conserved, whereas others are eliminated (2). The selected sequences are then extracted (3) and amplified by PCR (or RT-PCR followed by in vitro transcription for RNA libraries) (4). During this step, mutations can appear (5). The new library enters a new cycle of selection-amplification. Over the successive cycles, sequences that provide the best binding to the target are amplified and can be identified by sequencing (6).

Figure 4. In vivo studies evaluating the use of aptamers to design imaging probes or theranostic agents. A) Repartition of studies over time by imaging modality. B) Repartition of studies over time by imaging modality. C) Repartition of studies by disease model. D) Repartition of studies by target. 


\section{References and Notes}

[1] C. Tuerk, L. Gold, Systematic evolution of ligands by exponential enrichment : RNA ligands to bacteriophage T4 DNA polymerase, Science (New York, N.Y.), 249 (1990) 505-510.

[2] A.D. Ellington, J.W. Szostak, In vitro selection of RNA molecules that bind specific ligands., Nature, 346 (1990) 818-822.

[3] L. Gold, B. Polisky, O. Uhlenbeck, M. Yarus, Diversity of oligonucleotide functions, Annual review of biochemistry, 64 (1995) 763-797.

[4] T. Fitzwater, B. Polisky, A SELEX primer, Methods Enzymol, 267 (1996) 275-301.

[5] S.E. Osborne, A.D. Ellington, Nucleic Acid Selection and the Challenge of Combinatorial Chemistry, Chemical reviews, 97 (1997) 349-370.

[6] S.C. Gopinath, Methods developed for SELEX, Anal Bioanal Chem, 387 (2007) 171-182.

[7] A. Cibiel, D.M. Dupont, F. Duconge, Methods To Identify Aptamers against Cell Surface Biomarkers, Pharmaceuticals, 4 (2011) 1216-1235.

[8] M.C. Mercier, M. Dontenwill, L. Choulier, Selection of Nucleic Acid Aptamers Targeting Tumor Cell-Surface Protein Biomarkers, Cancers, 9 (2017).

[9] N. Nguyen Quang, G. Perret, F. Ducongé, Applications of High-Throughput Sequencing for In Vitro Selection and Characterization of Aptamers, Pharmaceuticals, 9 (2016) 76.

[10] R.R. White, B.A. Sullenger, C.P. Rusconi, Developing aptamers into therapeutics, J Clin Invest, 106 (2000) 929-934.

[11] S.J. Ni, H.Z. Yao, L.L. Wang, J. Lu, F. Jiang, A.P. Lu, G. Zhang, Chemical Modifications of Nucleic Acid Aptamers for Therapeutic Purposes, International journal of molecular sciences, 18 (2017).

[12] C. Pestourie, B. Tavitian, F. Duconge, Aptamers against extracellular targets for in vivo applications, Biochimie, 87 (2005) 921-930.

[13] A. Cibiel, C. Pestourie, F. Duconge, In vivo uses of aptamers selected against cell surface biomarkers for therapy and molecular imaging, Biochimie, 94 (2012) 1595-1606.

[14] P.K.R. Kumar, A.D. Ellington, Artificial evolution and natural ribozymes, FASEB journal : official publication of the Federation of American Societies for Experimental Biology, 9 (1995) 11831195.

[15] R.R. Breaker, G.F. Joyce, The expanding view of RNA and DNA function, Chem Biol, 21 (2014) 1059-1065.

[16] F. Pfeiffer, G. Mayer, Selection and Biosensor Application of Aptamers for Small Molecules, Front Chem, 4 (2016) 25.

[17] C. Forier, E. Boschetti, M. Ouhammouch, A. Cibiel, F. Duconge, M. Nogre, M. Tellier, D. Bataille, N. Bihoreau, P. Santambien, S. Chtourou, G. Perret, DNA aptamer affinity ligands for highly selective purification of human plasma-related proteins from multiple sources, J Chromatogr A, 1489 (2017) 39-50.

[18] M.V. Berezovski, M. Lechmann, M.U. Musheev, T.W. Mak, S.N. Krylov, Aptamer-Facilitated Biomarker Discovery (AptaBiD), J Am Chem Soc, (2008).

[19] C. Berens, B. Suess, Riboswitch engineering - making the all-important second and third steps, Curr Opin Biotechnol, 31C (2014) 10-15.

[20] J. Zhou, J. Rossi, Aptamers as targeted therapeutics: current potential and challenges, Nat Rev Drug Discov, (2016).

[21] S. Missailidis, A. Perkins, Update: aptamers as novel radiopharmaceuticals: their applications and future prospects in diagnosis and therapy, Cancer Biother Radiopharm, 22 (2007) 453-468.

[22] H. Hong, S. Goel, Y. Zhang, W. Cai, Molecular imaging with nucleic acid aptamers, Current medicinal chemistry, 18 (2011) 4195-4205.

[23] A.Z. Wang, O.C. Farokhzad, Current Progress of Aptamer-Based Molecular Imaging, Journal of Nuclear Medicine, (2014). 
[24] C.A. Dougherty, W. Cai, H. Hong, Applications of aptamers in targeted imaging: state of the art, Curr Top Med Chem, 15 (2015) 1138-1152.

[25] B. Tavitian, F. Duconge, R. Boisgard, F. Dolle, In vivo imaging of oligonucleotidic aptamers, Methods Mol Biol, 535 (2009) 241-259.

[26] M. Gijs, A. Aerts, N. Impens, S. Baatout, A. Luxen, Aptamers as radiopharmaceuticals for nuclear imaging and therapy, Nucl Med Biol, 43 (2016) 253-271.

[27] J. Charlton, J. Sennello, D. Smith, In vivo imaging of inflammation using an aptamer inhibitor of human neutrophil elastase, Chem Biol, 4 (1997) 809-816.

[28] B.J. Hicke, A.W. Stephens, T. Gould, Y.F. Chang, C.K. Lynott, J. Heil, S. Borkowski, C.S. Hilger, G. Cook, S. Warren, P.G. Schmidt, Tumor targeting by an aptamer, J Nucl Med, 47 (2006) 668-678.

[29] C.D. Pieve, A.C. Perkins, S. Missailidis, Anti-MUC1 aptamers: radiolabelling with (99m)Tc and biodistribution in MCF-7 tumour-bearing mice, Nucl Med Biol, 36 (2009) 703-710.

[30] Z. Noaparast, S.J. Hosseinimehr, M. Piramoon, S.M. Abedi, Tumor targeting with a (99m)Tclabeled AS1411 aptamer in prostate tumor cells, J Drug Target, 23 (2015) 497-505.

[31] K. Varmira, S.J. Hosseinimehr, Z. Noaparast, S.M. Abedi, A HER2-targeted RNA aptamer molecule labeled with $99 \mathrm{mTc}$ for single-photon imaging in malignant tumors, Nuclear Medicine and Biology, (2013).

[32] X. Wu, H. Liang, Y. Tan, C. Yuan, S. Li, X. Li, G. Li, Y. Shi, X. Zhang, Cell-SELEX aptamer for highly specific radionuclide molecular imaging of glioblastoma in vivo, PloS one, 9 (2014) e90752.

[33] S. Da Rocha Gomes, J. Miguel, L. Azema, S. Eimer, C. Ries, E. Dausse, H. Loiseau, M. Allard, J.J. Toulme, (99m)Tc-MAG3-Aptamer for Imaging Human Tumors Associated with High Level of Matrix Metalloprotease-9, Bioconjug Chem, (2012).

[34] D. Kryza, F. Debordeaux, L. Azema, A. Hassan, O. Paurelle, J. Schulz, C. Savona-Baron, E. Charignon, P. Bonazza, J. Taleb, P. Fernandez, M. Janier, J.J. Toulme, Ex Vivo and In Vivo Imaging and Biodistribution of Aptamers Targeting the Human Matrix MetalloProtease-9 in Melanomas, PloS one, 11 (2016) e0149387.

[35] C.M. de Sousa Lacerda, I.M. Ferreira, S.R. Dos Santos, A.L. de Barros, S.O. Fernandes, V.N. Cardoso, A.S. de Andrade, (1-->3)-beta-D-glucan aptamers labeled with technetium-99m: Biodistribution and imaging in experimental models of bacterial and fungal infection, Nucl Med Biol, 46 (2017) 19-24.

[36] I.M. Ferreira, C.M. de Sousa Lacerda, S.R. Dos Santos, A.L.B. de Barros, S.O. Fernandes, V.N. Cardoso, A.S.R. de Andrade, Detection of bacterial infection by a technetium-99m-labeled peptidoglycan aptamer, Biomedicine $\&$ pharmacotherapy = Biomedecine $\&$ pharmacotherapie, 93 (2017) 931-938.

[37] S.R.D. Santos, C.M. de Sousa Lacerda, I.M. Ferreira, A.L.B. de Barros, S.O. Fernandes, V.N. Cardoso, A.S.R. de Andrade, Scintigraphic imaging of Staphylococcus aureus infection using 99mTc radiolabeled aptamers, Applied radiation and isotopes : including data, instrumentation and methods for use in agriculture, industry and medicine, 128 (2017) 22-27.

[38] J. Li, H. Zheng, P.J. Bates, T. Malik, X.F. Li, J.O. Trent, C.K. Ng, Aptamer imaging with Cu-64 labeled AS1411: preliminary assessment in lung cancer, Nucl Med Biol, 41 (2014) 179-185.

[39] J.Y. Park, T.S. Lee, I.H. Song, Y.L. Cho, J.R. Chae, M. Yun, H. Kang, J.H. Lee, J.H. Lim, W.G. Cho, W.J. Kang, Hybridization-based aptamer labeling using complementary oligonucleotide platform for PET and optical imaging, Biomaterials, 100 (2016) 143-151.

[40] O. Jacobson, X. Yan, N. Gang, I.D. Weiss, Y. Ma, L.P. Szajek, B. Shen, D.O. Kiesewetter, X. Chen, PET Imaging of Tenascin-C with a Radiolabeled Single-Strand DNA Aptamer, Journal of Nuclear Medicine, (2015).

[41] D.A. Daniels, H. Chen, B.J. Hicke, K.M. Swiderek, L. Gold, A tenascin-C aptamer identified by tumor cell SELEX: systematic evolution of ligands by exponential enrichment, Proceedings of the National Academy of Sciences of the United States of America, 100 (2003) 15416-15421. 
[42] O. Jacobson, I.D. Weiss, L. Wang, Z. Wang, X. Yang, A. Dewhurst, Y. Ma, G. Zhu, G. Niu, D.O. Kiesewetter, N. Vasdev, S.H. Liang, X. Chen, 18F-Labeled Single-Stranded DNA Aptamer for PET Imaging of Protein Tyrosine Kinase-7 Expression, J Nucl Med, 56 (2015) 1780-1785.

[43] I. Theodorou, N.N. Quang, K. Gombert, B. Theze, B. Lelandais, F. Duconge, In Vitro and In Vivo Imaging of Fluorescent Aptamers, Methods Mol Biol, 1380 (2016) 135-150.

[44] H. Shi, Z. Tang, Y. Kim, H. Nie, Y.F. Huang, X. He, K. Deng, K. Wang, W. Tan, In vivo fluorescence imaging of tumors using molecular aptamers generated by cell-SELEX, Chem Asian J, 5 (2010) 2209-2213.

[45] P. Gong, B. Shi, M. Zheng, B. Wang, P. Zhang, D. Hu, D. Gao, Z. Sheng, C. Zheng, Y. Ma, L. Cai, PEI protected aptamer molecular probes for contrast-enhanced in vivo cancer imaging, Biomaterials, 33 (2012) 7810-7817.

[46] A. Cibiel, N.N. Quang, K. Gombert, B. Theze, A. Garofalakis, F. Duconge, From ugly duckling to swan: unexpected identification from cell-SELEX of an anti-Annexin A2 aptamer targeting tumors, PloS one, 9 (2014) e87002.

[47] H. Shi, X. He, W. Cui, K. Wang, K. Deng, D. Li, F. Xu, Locked nucleic acid/DNA chimeric aptamer probe for tumor diagnosis with improved serum stability and extended imaging window in vivo, Anal Chim Acta, 812 (2014) 138-144.

[48] V. Calzada, M. Moreno, J. Newton, J. Gonzalez, M. Fernandez, J.P. Gambini, M. Ibarra, A. Chabalgoity, S. Deutscher, T. Quinn, P. Cabral, H. Cerecetto, Development of new PTK7-targeting aptamer-fluorescent and -radiolabelled probes for evaluation as molecular imaging agents: Lymphoma and melanoma in vivo proof of concept, Bioorganic \& medicinal chemistry, 25 (2017) 1163-1171.

[49] A. Garofalakis, A. Dubois, B. Kuhnast, D.M. Dupont, I. Janssens, N. Mackiewicz, F. Dolle, B. Tavitian, F. Duconge, In vivo validation of free-space fluorescence tomography using nuclear imaging, Optics letters, 35 (2010) 3024-3026.

[50] J. Mi, Y. Liu, Z.N. Rabbani, Z. Yang, J.H. Urban, B.A. Sullenger, B.M. Clary, In vivo selection of tumor-targeting RNA motifs, Nature chemical biology, 6 (2009) 22-24.

[51] H. Shi, X. He, K. Wang, X. Wu, X. Ye, Q. Guo, W. Tan, Z. Qing, X. Yang, B. Zhou, Activatable aptamer probe for contrast-enhanced in vivo cancer imaging based on cell membrane protein-triggered conformation alteration, Proceedings of the National Academy of Sciences of the United States of America, 108 (2011) 3900-3905.

[52] C. Li, Y. Meng, S. Wang, M. Qian, J. Wang, W. Lu, R. Huang, Mesoporous Carbon Nanospheres Featured Fluorescent Aptasensor for Multiple Diagnosis of Cancer in Vitro and in Vivo, ACS Nano, 9 (2015) 12096-12103.

[53] C.T. Farrar, C.M. William, E. Hudry, T. Hashimoto, B.T. Hyman, RNA aptamer probes as optical imaging agents for the detection of amyloid plaques, PloS one, 9 (2014) e89901.

[54] T. Simao, A. Ng, D. Fatehi, S. Corluka, A. Abulrob, M. Zourob, Development of an AntiVascular Cell Adhesion Protein-1 Aptamer for Molecular Imaging and Inflammation Detection in Transgenic Mouse Model of Alzheimer's Disease, J Biomed Nanotechnol, 11 (2015) 2264-2274.

[55] H. Hu, A. Dai, J. Sun, X. Li, F. Gao, L. Wu, Y. Fang, H. Yang, L. An, H. Wu, S. Yang, Aptamerconjugated $\mathrm{Mn} 3 \mathrm{O} 4 @ \mathrm{SiO} 2$ core-shell nanoprobes for targeted magnetic resonance imaging, Nanoscale, 5 (2013) 10447-10454.

[56] B. Kim, J. Yang, M. Hwang, J. Choi, H.O. Kim, E. Jang, J.H. Lee, S.H. Ryu, J.S. Suh, Y.M. Huh, S. Haam, Aptamer-modified magnetic nanoprobe for molecular MR imaging of VEGFR2 on angiogenic vasculature, Nanoscale research letters, 8 (2013) 399.

[57] E.K. Lim, B. Kim, Y. Choi, Y. Ro, E.J. Cho, J.H. Lee, S.H. Ryu, J.S. Suh, S. Haam, Y.M. Huh, Aptamer-conjugated magnetic nanoparticles enable efficient targeted detection of integrin alphavbeta3 via magnetic resonance imaging, Journal of biomedical materials research. Part A, 102 (2014) 49-59.

[58] X.G. You, R. Tu, M.L. Peng, Y.J. Bai, M. Tan, H.J. Li, J. Guan, L.J. Wen, Molecular magnetic resonance probe targeting VEGF165: preparation and in vitro and in vivo evaluation, Contrast media \& molecular imaging, 9 (2014) 349-354. 
[59] M.A. Nakatsuka, C.V. Barback, K.R. Fitch, A.R. Farwell, S.C. Esener, R.F. Mattrey, J.N. Cha, A.P. Goodwin, In vivo ultrasound visualization of non-occlusive blood clots with thrombin-sensitive contrast agents, Biomaterials, 34 (2013) 9559-9565.

[60] W. Hwang do, H.Y. Ko, J.H. Lee, H. Kang, S.H. Ryu, I.C. Song, D.S. Lee, S. Kim, A nucleolintargeted multimodal nanoparticle imaging probe for tracking cancer cells using an aptamer, J Nucl Med, 51 (2010) 98-105.

[61] C.H. Li, T.R. Kuo, H.J. Su, W.Y. Lai, P.C. Yang, J.S. Chen, D.Y. Wang, Y.C. Wu, C.C. Chen, Fluorescence-Guided Probes of Aptamer-Targeted Gold Nanoparticles with Computed Tomography Imaging Accesses for in Vivo Tumor Resection, Sci Rep, 5 (2015) 15675.

[62] Z. Xiao, O.C. Farokhzad, Aptamer-functionalized nanoparticles for medical applications: challenges and opportunities, ACS Nano, 6 (2012) 3670-3676.

[63] L. Pascual, C. Cerqueira-Coutinho, A. Garcia-Fernandez, B. de Luis, E.S. Bernardes, M.S. Albernaz, S. Missailidis, R. Martinez-Manez, R. Santos-Oliveira, M. Orzaez, F. Sancenon, MUC1 aptamer-capped mesoporous silica nanoparticles for controlled drug delivery and radio-imaging applications, Nanomedicine, 13 (2017) 2495-2505.

[64] J. Mosafer, K. Abnous, M. Tafaghodi, A. Mokhtarzadeh, M. Ramezani, In vitro and in vivo evaluation of anti-nucleolin-targeted magnetic PLGA nanoparticles loaded with doxorubicin as a theranostic agent for enhanced targeted cancer imaging and therapy, European journal of pharmaceutics and biopharmaceutics : official journal of Arbeitsgemeinschaft fur Pharmazeutische Verfahrenstechnik e.V, 113 (2017) 60-74.

[65] J. Mosafer, M. Teymouri, K. Abnous, M. Tafaghodi, M. Ramezani, Study and evaluation of nucleolin-targeted delivery of magnetic PLGA-PEG nanospheres loaded with doxorubicin to C6 glioma cells compared with low nucleolin-expressing L929 cells, Materials science \& engineering. C, Materials for biological applications, 72 (2017) 123-133.

[66] R. Savla, O. Taratula, O. Garbuzenko, T. Minko, Tumor targeted quantum dot-mucin 1 aptamerdoxorubicin conjugate for imaging and treatment of cancer, J Control Release, 153 (2011) 16-22.

[67] D. Chen, B. Li, S. Cai, P. Wang, S. Peng, Y. Sheng, Y. He, Y. Gu, H. Chen, Dual targeting luminescent gold nanoclusters for tumor imaging and deep tissue therapy, Biomaterials, 100 (2016) 116.

[68] M.K. Yu, D. Kim, I.H. Lee, J.S. So, Y.Y. Jeong, S. Jon, Image-guided prostate cancer therapy using aptamer-functionalized thermally cross-linked superparamagnetic iron oxide nanoparticles, Small, 7 (2011) 2241-2249.

[69] S.H. Jalalian, S.M. Taghdisi, N. Shahidi Hamedani, S.A. Kalat, P. Lavaee, M. Zandkarimi, N. Ghows, M.R. Jaafari, S. Naghibi, N.M. Danesh, M. Ramezani, K. Abnous, Epirubicin loaded super paramagnetic iron oxide nanoparticle-aptamer bioconjugate for combined colon cancer therapy and imaging in vivo, European journal of pharmaceutical sciences : official journal of the European Federation for Pharmaceutical Sciences, 50 (2013) 191-197.

[70] Y. Lei, J. Tang, H. Shi, X. Ye, X. He, F. Xu, L. Yan, Z. Qiao, K. Wang, Nature-Inspired Smart DNA Nanodoctor for Activatable In Vivo Cancer Imaging and In Situ Drug Release Based on Recognition-Triggered Assembly of Split Aptamer, Anal Chem, 88 (2016) 11699-11706.

[71] W. Tao, X. Zeng, J. Wu, X. Zhu, X. Yu, X. Zhang, J. Zhang, G. Liu, L. Mei, PolydopamineBased Surface Modification of Novel Nanoparticle-Aptamer Bioconjugates for In Vivo Breast Cancer Targeting and Enhanced Therapeutic Effects, Theranostics, 6 (2016) 470-484.

[72] Z. Luo, Z. Yan, K. Jin, Q. Pang, T. Jiang, H. Lu, X. Liu, Z. Pang, L. Yu, X. Jiang, Precise glioblastoma targeting by AS1411 aptamer-functionalized poly (l-gamma-glutamylglutamine)paclitaxel nanoconjugates, Journal of colloid and interface science, 490 (2017) 783-796.

[73] H. Gao, J. Qian, S. Cao, Z. Yang, Z. Pang, S. Pan, L. Fan, Z. Xi, X. Jiang, Q. Zhang, Precise glioma targeting of and penetration by aptamer and peptide dual-functioned nanoparticles, Biomaterials, 33 (2012) 5115-5123. 
[74] H. Gao, J. Qian, Z. Yang, Z. Pang, Z. Xi, S. Cao, Y. Wang, S. Pan, S. Zhang, W. Wang, X. Jiang, Q. Zhang, Whole-cell SELEX aptamer-functionalised poly(ethyleneglycol)-poly(epsilon-caprolactone) nanoparticles for enhanced targeted glioblastoma therapy, Biomaterials, 33 (2012) 6264-6272.

[75] J.K. Kim, K.-J. Choi, M. Lee, M.-h. Jo, S. Kim, Molecular imaging of a cancer-targeting theragnostics probe using a nucleolin aptamer- and microRNA-221 molecular beacon-conjugated nanoparticle, Biomaterials, (2011).

[76] J. Tian, L. Ding, H. Ju, Y. Yang, X. Li, Z. Shen, Z. Zhu, J.S. Yu, C.J. Yang, A multifunctional nanomicelle for real-time targeted imaging and precise near-infrared cancer therapy, Angew Chem Int Ed Engl, 53 (2014) 9544-9549.

[77] X. Ye, H. Shi, X. He, Y. Yu, D. He, J. Tang, Y. Lei, K. Wang, Cu-Au alloy nanostructures coated with aptamers: a simple, stable and highly effective platform for in vivo cancer theranostics, Nanoscale, 8 (2016) 2260-2267.

[78] H. Shi, X. Ye, X. He, K. Wang, W. Cui, D. He, D. Li, X. Jia, Au@Ag/Au nanoparticles assembled with activatable aptamer probes as smart "nano-doctors" for image-guided cancer thermotherapy, Nanoscale, 6 (2014) 8754-8761.

[79] E.Y. Chuang, C.C. Lin, K.J. Chen, D.H. Wan, K.J. Lin, Y.C. Ho, P.Y. Lin, H.W. Sung, A FRETguided, NIR-responsive bubble-generating liposomal system for in vivo targeted therapy with spatially and temporally precise controlled release, Biomaterials, 93 (2016) 48-59.

[80] F. Zhao, J. Zhou, X. Su, Y. Wang, X. Yan, S. Jia, B. Du, A Smart Responsive Dual AptamersTargeted Bubble-Generating Nanosystem for Cancer Triplex Therapy and Ultrasound Imaging, Small, 13 (2017).

[81] K. Roy, R.K. Kanwar, J.R. Kanwar, LNA aptamer based multi-modal, Fe3O4-saturated lactoferrin (Fe3O4-bLf) nanocarriers for triple positive (EpCAM, CD133, CD44) colon tumor targeting and NIR, MRI and CT imaging, Biomaterials, 71 (2015) 84-99.

[82] H. Tsuda, T. Kozu, G. Iinuma, Y. Ohashi, Y. Saito, D. Saito, T. Akasu, D.B. Alexander, M. Futakuchi, K. Fukamachi, J. Xu, T. Kakizoe, M. Iigo, Cancer prevention by bovine lactoferrin: from animal studies to human trial, Biometals : an international journal on the role of metal ions in biology, biochemistry, and medicine, 23 (2010) 399-409.

[83] A. Bouvier-Muller, F. Duconge, Nucleic acid aptamers for neurodegenerative diseases, Biochimie, 145 (2018) 73-83.

[84] K.S. Park, Nucleic acid aptamer-based methods for diagnosis of infections, Biosens Bioelectron, 102 (2018) 179-188.

[85] M. Avci-Adali, H. Steinle, T. Michel, C. Schlensak, H.P. Wendel, Potential Capacity of Aptamers to Trigger Immune Activation in Human Blood, PloS one, 8 (2013).

[86] H. Maeda, G.Y. Bharate, J. Daruwalla, Polymeric drugs for efficient tumor-targeted drug delivery based on EPR-effect, European journal of pharmaceutics and biopharmaceutics : official journal of Arbeitsgemeinschaft fur Pharmazeutische Verfahrenstechnik e.V, 71 (2009) 409-419.

[87] H. Dougan, J.I. Weitz, A.R. Stafford, K.D. Gillespie, P. Klement, J.B. Hobbs, D.M. Lyster, Evaluation of DNA aptamers directed to thrombin as potential thrombus imaging agents, Nucl Med Biol, 30 (2003) 61-72.

[88] M.A. Nakatsuka, R.F. Mattrey, S.C. Esener, J.N. Cha, A.P. Goodwin, Aptamer-Crosslinked Microbubbles: Smart Contrast Agents for Thrombin-Activated Ultrasound Imaging, Advanced materials (Deerfield Beach, Fla.), (2012).

[89] H. Zhang, 99mTc-Mercaptoacetyl-Glu-Glu-aptamer specific for tenascin-C, in: Molecular Imaging and Contrast Agent Database (MICAD), Bethesda MD, 2004.

[90] K.E. Borbas, C.S. Ferreira, A. Perkins, J.I. Bruce, S. Missailidis, Design and synthesis of monoand multimeric targeted radiopharmaceuticals based on novel cyclen ligands coupled to anti-MUC1 aptamers for the diagnostic imaging and targeted radiotherapy of cancer, Bioconjug Chem, 18 (2007) 1205-1212. 
[91] C. Zhang, X. Ji, Y. Zhang, G. Zhou, X. Ke, H. Wang, P. Tinnefeld, Z. He, One-pot synthesized aptamer-functionalized CdTe:Zn2+ quantum dots for tumor-targeted fluorescence imaging in vitro and in vivo, Anal Chem, 85 (2013) 5843-5849.

[92] K. Varmira, S.J. Hosseinimehr, Z. Noaparast, S.M. Abedi, An improved radiolabelled RNA aptamer molecule for HER2 imaging in cancers, J Drug Target, 22 (2014) 116-122.

[93] L. Wang, O. Jacobson, D. Avdic, B.H. Rotstein, I.D. Weiss, L. Collier, X. Chen, N. Vasdev, S.H. Liang, Ortho-Stabilized (18) F-Azido Click Agents and their Application in PET Imaging with SingleStranded DNA Aptamers, Angew Chem Int Ed Engl, 54 (2015) 12777-12781.

[94] M. Yi, S. Yang, Z. Peng, C. Liu, J. Li, W. Zhong, R. Yang, W. Tan, Two-photon graphene oxide/aptamer nanosensing conjugate for in vitro or in vivo molecular probing, Anal Chem, 86 (2014) 3548-3554.

[95] Z. Zeng, P. Parekh, Z. Li, Z.Z. Shi, C.H. Tung, Y. Zu, Specific and sensitive tumor imaging using biostable oligonucleotide aptamer probes, Theranostics, 4 (2014) 945-952.

[96] F. Ding, S. Guo, M. Xie, W. Luo, C. Yuan, W. Huang, Y. Zhou, X.L. Zhang, X. Zhou, Diagnostic applications of gastric carcinoma cell aptamers in vitro and in vivo, Talanta, 134 (2015) 30-36.

[97] J. Tang, N. Huang, X. Zhang, T. Zhou, Y. Tan, J. Pi, L. Pi, S. Cheng, H. Zheng, Y. Cheng, Aptamer-conjugated PEGylated quantum dots targeting epidermal growth factor receptor variant III for fluorescence imaging of glioma, Int J Nanomedicine, 12 (2017) 3899-3911.

[98] L. Tang, X. Yang, L.W. Dobrucki, I. Chaudhury, Q. Yin, C. Yao, S. Lezmi, W.G. Helferich, T.M. Fan, J. Cheng, Aptamer-functionalized, ultra-small, monodisperse silica nanoconjugates for targeted dual-modal imaging of lymph nodes with metastatic tumors, Angew Chem Int Ed Engl, 51 (2012) 12721-12726.

[99] X. Fan, Y. Guo, L. Wang, X. Xiong, L. Zhu, K. Fang, Diagnosis of prostate cancer using antiPSMA aptamer A10-3.2-oriented lipid nanobubbles, Int J Nanomedicine, 11 (2016) 3939-3950.

[100] Z. Wei, Y. Wu, Y. Zhao, L. Mi, J. Wang, J. Wang, J. Zhao, L. Wang, A. Liu, Y. Li, W. Wei, Y. Zhang, S. Liu, Multifunctional nanoprobe for cancer cell targeting and simultaneous fluorescence/magnetic resonance imaging, Anal Chim Acta, 938 (2016) 156-164.

[101] H. Zhu, L. Zhang, Y. Liu, Y. Zhou, K. Wang, X. Xie, L. Song, D. Wang, C. Han, Q. Chen, Aptamer-PEG-modified Fe3O4@Mn as a novel T1- and T2- dual-model MRI contrast agent targeting hypoxia-induced cancer stem cells, Sci Rep, 6 (2016) 39245.

[102] C.H. Liu, J. Ren, C.M. Liu, P.K. Liu, Intracellular gene transcription factor protein-guided MRI by DNA aptamers in vivo, FASEB journal : official publication of the Federation of American Societies for Experimental Biology, 28 (2014) 464-473.

[103] L. Shan, Indotricarbocyanine-loaded AS1411 DNA aptamer- and TGN peptide-modified poly(ethylene glycol)-poly(epsilon-caprolactone) nanoparticles, in: Molecular Imaging and Contrast Agent Database (MICAD), Bethesda MD, 2004.

[104] F. Santos do Carmo, E. Ricci-Junior, C. Cerqueira-Coutinho, M.S. Albernaz, E.S. Bernardes, S. Missailidis, R. Santos-Oliveira, Anti-MUC1 nano-aptamers for triple-negative breast cancer imaging by single-photon emission computed tomography in inducted animals: initial considerations, Int $\mathbf{J}$ Nanomedicine, 12 (2017) 53-60.

[105] C. Li, M. Qian, S. Wang, H. Jiang, Y. Du, J. Wang, W. Lu, N. Murthy, R. Huang, Aptavalvegated Mesoporous Carbon Nanospheres image Cellular Mucin and provide On-demand Targeted Drug Delivery, Theranostics, 7 (2017) 3319-3325.

[106] J. Gao, C. Wu, D. Deng, P. Wu, C. Cai, Direct Synthesis of Water-Soluble Aptamer-Ag2 S Quantum Dots at Ambient Temperature for Specific Imaging and Photothermal Therapy of Cancer, Advanced healthcare materials, 5 (2016) 2437-2449.

[107] G. Zhu, J. Zheng, E. Song, M. Donovan, K. Zhang, C. Liu, W. Tan, Self-assembled, aptamertethered DNA nanotrains for targeted transport of molecular drugs in cancer theranostics, Proceedings of the National Academy of Sciences of the United States of America, 110 (2013) 7998-8003.

[108] M.P. Melancon, M. Zhou, R. Zhang, C. Xiong, P. Allen, X. Wen, Q. Huang, M. Wallace, J.N. Myers, R.J. Stafford, D. Liang, A.D. Ellington, C. Li, Selective uptake and imaging of aptamer- and 
antibody-conjugated hollow nanospheres targeted to epidermal growth factor receptors overexpressed in head and neck cancer, ACS Nano, 8 (2014) 4530-4538.

[109] M.W. Kim, H.Y. Jeong, S.J. Kang, M.J. Choi, Y.M. You, C.S. Im, T.S. Lee, I.H. Song, C.G. Lee, K.J. Rhee, Y.K. Lee, Y.S. Park, Cancer-targeted Nucleic Acid Delivery and Quantum Dot Imaging Using EGF Receptor Aptamer-conjugated Lipid Nanoparticles, Sci Rep, 7 (2017) 9474.

[110] D. Zhang, A. Zheng, J. Li, M. Wu, L. Wu, Z. Wei, N. Liao, X. Zhang, Z. Cai, H. Yang, G. Liu, X. Liu, J. Liu, Smart Cu(II)-aptamer complexes based gold nanoplatform for tumor microenvironment triggered programmable intracellular prodrug release, photodynamic treatment and aggregation induced photothermal therapy of hepatocellular carcinoma, Theranostics, 7 (2017) 164179. 


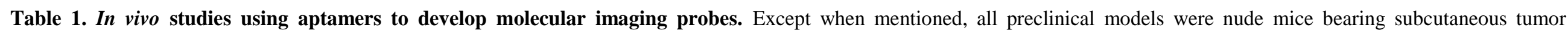
xenografts $(S$.) or orthotopic tumor xenografts (O.).

\begin{tabular}{|c|c|c|c|c|c|c|}
\hline Target & Name & Chemistry & Imaging & Labeling & Preclinical model & References \\
\hline Neutrophil elastase & NX21909 & DNA & SPECT & ${ }^{99 \mathrm{~m}} \mathrm{Tc}$ & $\begin{array}{l}\text { Artificially induced inflammation in male Harlan } \\
\text { Sprague-Dawley rats }\end{array}$ & [27] \\
\hline Thrombin & ODN1\& ODN2 & DNA & $\begin{array}{l}\text { Scintigraphy } \\
\text { (SPECT) }\end{array}$ & ${ }^{123} \mathrm{I}$ & $\begin{array}{l}\text { Rabbit jugular vein model using thrombus } \\
\text { supplemented with human thrombin }\end{array}$ & [87] \\
\hline Thrombin & & DNA & Ultrasound & Microbubbles & Rabbit Deep Venous Thrombosis Model & {$[59,88]$} \\
\hline Tenascin-C & TTA1 & $\begin{array}{l}\text { 2'OMe-Pu, } \\
\text { 2'F-Py RNA }\end{array}$ & SPECT & ${ }^{99 \mathrm{~m}} \mathrm{Tc}$ & S. (U251 cells) and O. (MDA-MB-435 cells) & [89] [28] \\
\hline Tenascin-C & GBI-10 & DNA & PET & ${ }^{18} \mathrm{~F}$ and ${ }^{64} \mathrm{Cu}$ & $\begin{array}{l}\text { S. (U87MG cells or H460) and O. (MDA-MB- } \\
435)\end{array}$ & {$[40]$} \\
\hline MUC1 & MUC1 & DNA & $\begin{array}{l}\text { Ex vivo } \\
\text { Scintigraphy }\end{array}$ & $99 \mathrm{mTc}$ & S. (MCF-7 cells) & [90] \\
\hline MUC1 & & Modified DNA & Fluorescence & Quantum Dots & S. (A549 cells) & [91] \\
\hline MUC1 & P0 & DNA & Fluorescence & Су3 & S. (MCF-7 cells) & {$[52]$} \\
\hline Nucleolin & AS1411 & DNA & PET & ${ }^{64} \mathrm{Cu}$ & S. (H460 cells) & [38] \\
\hline Nucleolin & AS1411 & DNA & SPECT & ${ }^{99 \mathrm{~m}} \mathrm{Tc}$ & S. (DU-145 cells) & [30] \\
\hline Nucleolin & AS1411 & DNA & MRI & Magnetic NPs & S. (HeLa cells) & [55] \\
\hline IGHM & TD05 & DNA & Fluorescence & Cy5 & S. (Ramos cells) & {$[44]$} \\
\hline IGHM & TD05 & DNA & Fluorescence & Cy5 FITC & S. (Ramos cells) & [45] \\
\hline IGHM & TD05 & DNA & Fluorescence & Cy5 & S. (Ramos cells) & {$[47]$} \\
\hline $\begin{array}{l}\text { ErbB2-expressing } \\
\text { breast cancer cell line }\end{array}$ & HER2 & 2'F-Py RNA & SPECT & ${ }^{99 \mathrm{~m}} \mathrm{Tc}$ & S. (SKOV-3 cells) & {$[31,92]$} \\
\hline
\end{tabular}




\begin{tabular}{|c|c|c|c|c|c|c|}
\hline EGFRvIII & $\mathrm{U} 2$ & DNA & SPECT & ${ }^{188} \operatorname{Re}$ & S. (U87MG cells) & {$[32]$} \\
\hline PTK7 & $\operatorname{Sgc} 8$ & DNA & Fluorescence & Cy5 & S. (CCRF-CEM cells) & {$[51]$} \\
\hline PTK7 & Sgc8 & DNA & PET & ${ }^{18} \mathrm{~F}$ & $\begin{array}{l}\text { S. (U87MG cells or HCT116 cells) and tumor } \\
\text { metastases (HCT116 cells) }\end{array}$ & {$[40,93]$} \\
\hline$(1 \rightarrow 3)$ - $\beta$-D-glucan & seq6 \& seq30 & DNA & SPECT & ${ }^{99 \mathrm{~m}} \mathrm{Tc}$ & $\begin{array}{l}\text { Mice infected with S. aureus or } \\
\text { immunosuppressed and infected with } \text { C. albicans }\end{array}$ & [35] \\
\hline Peptidoglycan & Antibac1 & DNA & SPECT & ${ }^{99 \mathrm{~m}} \mathrm{Tc}$ & Mice infected with $S$. aureus or C. albicans & {$[36]$} \\
\hline $\begin{array}{l}\text { Staphylococcus } \\
\text { aureus }\end{array}$ & SA20, SA23 and SA34 & DNA & SPECT & ${ }^{99 \mathrm{~m}} \mathrm{Tc}$ & Mice infected with $S$. aureus & [37] \\
\hline MMP-9 & F3B & $\begin{array}{l}\text { 2’OMe-Pu, } \\
\text { 2'F-Py RNA }\end{array}$ & $\begin{array}{c}\text { SPECT / } \\
\text { Fluorescence }\end{array}$ & $\begin{array}{c}{ }^{99 \mathrm{~m}} \mathrm{Tc} \text { or }{ }^{111} \mathrm{In} / \\
\mathrm{Cy} 5\end{array}$ & S. (A375 cells) & {$[33,34]$} \\
\hline VEGFR2 & & BzdU DNA & MRI & Magnetic Nps & O. (U87MG cells) & [56] \\
\hline$\alpha v \beta 3$ Integrin & $\mathrm{Apt}_{\mathfrak{x} \mathrm{B} 33}$ & BzdU DNA & MRI & Magnetic Nps & S. (A431 cells) & [57] \\
\hline VEGF $_{165}$ & VEGF165-aptamer & $\begin{array}{l}\text { 2’OMe-Pu, } \\
\text { 2'F-Py RNA }\end{array}$ & MRI & USPIO & O. (BEL-7402 cells) & [58] \\
\hline $\mathrm{A} \beta$ fibrils & $\beta 55$ & RNA & Fluorescence & Fluorescein & APP/PS1 transgenic mice & [53] \\
\hline ATP & & DNA & Fluorescence & TPdye \& graphene oxide & Two-day-old zebrafish & [94] \\
\hline CD30 & & RNA \& DNA & Fluorescence & IRD800CW & Several subcutaneous tumor xenografts & [95] \\
\hline Annexin A2 & ACE4 & 2'F-Py RNA & Fluorescence & AF680 & S. (MCF-7 cells) & [46] \\
\hline SGC7901 cells & S4a & DNA & Fluorescence & $\begin{array}{l}\text { Quantum Dots / } \\
\text { Cy5/BHQ2 }\end{array}$ & S. (SGC7901 cells) & [96] \\
\hline VCAM-1 & & & Fluorescence & Cy5.5 & Transgenic APP/PS1 mice. & [54] \\
\hline EGFRvIII & A32 & DNA & Fluorescence & Quantum Dots & O. (U87MG cells) & [97] \\
\hline
\end{tabular}


PTK7

Sgc8-c

DNA

SPECT or

${ }^{99 \mathrm{~m}} \mathrm{Tc}$ or Alexa647 dye

S. (B16F1 cells and A20 cells)

[48]

Table 2. In vivo studies using aptamers to develop multimodal molecular imaging probes. Except when mentioned, all preclinical models were nude mice bearing subcutaneous tumor xenografts $(S$.) or orthotopic tumor xenografts (O.).

\begin{tabular}{|c|c|c|c|c|c|c|}
\hline Target & Name & Chemistry & Imaging & Labeling & Preclinical model & References \\
\hline Nucleolin & AS1411 & DNA & SPECT / Fluorescence / MRI & $\begin{array}{c}{ }^{67} \mathrm{Ga} / \text { rhodamine B / } \\
\text { Magnetic NPs }\end{array}$ & S. (C6 cells) & [60] \\
\hline Nucleolin & AS1411 & DNA & PET / Fluorescence & ${ }^{64} \mathrm{Cu} /$ Rhodamine B & S. (4T1 cells) & [98] \\
\hline Nucleolin & AS1411 & DNA & PET / Fluorescence & ${ }^{18} \mathrm{~F} / \mathrm{Cy} 5$ & S. (C6 cells) & [39] \\
\hline Nucleolin & AS1411 & DNA & $\mathrm{CT} /$ Fluorescence & Gold NPs / dye & S. (CL1-5 cells) & [61] \\
\hline PSMA & A10-3.2 & 2'F-Py RNA & Echography / Fluorescence & Nanobubbles / Cy5.5 & S. (PC-3 cells) & [99] \\
\hline TLS11a & Apt1 & DNA & MRI/ Fluorescence & Magnetic NPs / FITC & O. (HepG2 cells) & [100] \\
\hline HIF-1 $\alpha$ & & DNA & Dual MRI (T1\&T2) & Magnetic NPs & S. (Panc-1 cells) & [101] \\
\hline
\end{tabular}


Table 3. In vivo studies using aptamers to develop theranostic agents. Except when mentioned, all preclinical models were nude mice bearing subcutaneous tumor xenografts $(S$.) or orthotopic tumor xenografts (O.).

\begin{tabular}{|c|c|c|c|c|c|c|c|}
\hline Target & Name & Chemistry & Imaging & Labeling & Therapy & Preclinical model & References \\
\hline Nucleolin & AS1411 & DNA & Fluorescence & Dye & Doxorubicin & S. (U87MG cells) & [67] \\
\hline Nucleolin & AS1411 & DNA & Fluorescence & DiR & Docetaxel & O. (C6 cells) & [73] \\
\hline Nucleolin & AS1411 & DNA & Fluorescence & Molecular beacon & Antagomir & S. (C6 cells) & [75] \\
\hline Nucleolin & AS1411 & DNA & Fluorescence & DiR & Paclitaxel & O. (U87-PMT48-luc cells) & [72] \\
\hline Nucleolin & AS1411 & DNA & MRI & SPION & Doxorubicin & S. (C26 cells) & {$[64,65]$} \\
\hline Nucleolin & AS1411 & DNA & Fluorescence & DiR & Docetaxel & O. (C6 cells) & [103] \\
\hline Nucleolin & AS1411 & DNA & Fluorescence & IR-780 & Docetaxel & $\begin{array}{l}\text { S. (MCF-7 or MDA-MB-231 cells) and } \\
\text { spontaneous breast cancer bearing mice }\end{array}$ & [71] \\
\hline $\begin{array}{l}\text { Nucleolin } \\
\& \text { MUC1 }\end{array}$ & $\begin{array}{l}\text { AS1411 } \\
\& \text { S2.2 }\end{array}$ & DNA & Echography & & Docetaxel \& PTT & S. (S180 cells) & [80] \\
\hline MUC1 & & DNA & SPECT & ${ }^{99 \mathrm{~m}} \mathrm{Tc}$ & & S. (MDA-MB-231 cells) & [104] \\
\hline MUC1 & $\mathrm{P}_{0}$ & DNA & Fluorescence & Doxorubicin & Doxorubicin & S. (MCF-7 cells) & [105] \\
\hline MUC1 & MUC1 & DNA & Fluorescence & Су3 & Doxorubicin & S. (MCF-7 cells) & [79] \\
\hline MUC1 & S2.2 & DNA & Fluorescence & Quantum Dots & PTT & S. (MCF-7 cells) & [106] \\
\hline MUC1 & 5TR1 & DNA & MRI & SPION & Epirubicin & S. (C26 cells) & [69] \\
\hline
\end{tabular}




\begin{tabular}{|c|c|c|c|c|c|c|c|}
\hline MUC1 & & DNA & PET & ${ }^{99 \mathrm{~m}} \mathrm{Tc}$ & Doxorubicin & S. (MDA-MB-231 cells) & [63] \\
\hline MUC1 & MUC1 & DNA & Fluorescence & Quantum Dot & Doxorubicin & S. (A2780/AD cells) & [66] \\
\hline PTK7 & $\operatorname{Sgc} 8$ & DNA & Fluorescence & Doxorubicin & Doxorubicin & S. (CEM cells) & [107] \\
\hline PTK7 & $\mathrm{Sgc} 8 \mathrm{c}$ & DNA & Fluorescence & Сy5 & Doxorubicin & S. (CEM cells) & [70] \\
\hline PTK7 & $\mathrm{Sgc} 8 \mathrm{c}$ & DNA & Fluorescence & Cy5 & PTT & S. (CCRF-CEM cells) & [77] \\
\hline EGFR & & 2'F-Py RNA & SPECT & ${ }^{111} \mathrm{In}$ & $\varnothing$ & O. (OSC-19 cells) & [108] \\
\hline EGFR & & & Fluorescence & Quantum Dot & SiRNA & S. (MDA-MB-231 cells) & [109] \\
\hline EpCAM \& Nucleolin & & LNA & $\begin{array}{l}\text { Fluorescence/ } \\
\text { MRI / X-ray }\end{array}$ & $\begin{array}{l}\text { VivoTag } 680 \mathrm{XL} / \\
\mathrm{Fe}_{3} \mathrm{O}_{4}\end{array}$ & Bovine lactoferrin & S. (Caco- 2 cells) & [81] \\
\hline $\begin{array}{l}\text { Hepatocellular } \\
\text { carcinoma cells }\end{array}$ & TLS11a & ssDNA & Fluorescence & Chlorin e6 & PDT/PTT/AQ4N & S. (HepG2 cells) & [110] \\
\hline HER2 \& A549 cells & S6 & DNA & Fluorescence & Сy5 & PTT & S. (A549 cells) & [78] \\
\hline MDA-MB-231 cells & Apt S1 & DNA & Fluorescence & R16 FP & PDT & S. and O. (MDA-MB-231 cells) & [76] \\
\hline PSMA & A9 & RNA & MRI & SPION & Doxorubicin & S. (LNCaP cells) & [68] \\
\hline U87 cells & GMT8 & DNA & Fluorescence & DiR & Docetaxel & O. (U87 cells) & [74] \\
\hline
\end{tabular}


A

Figure 1

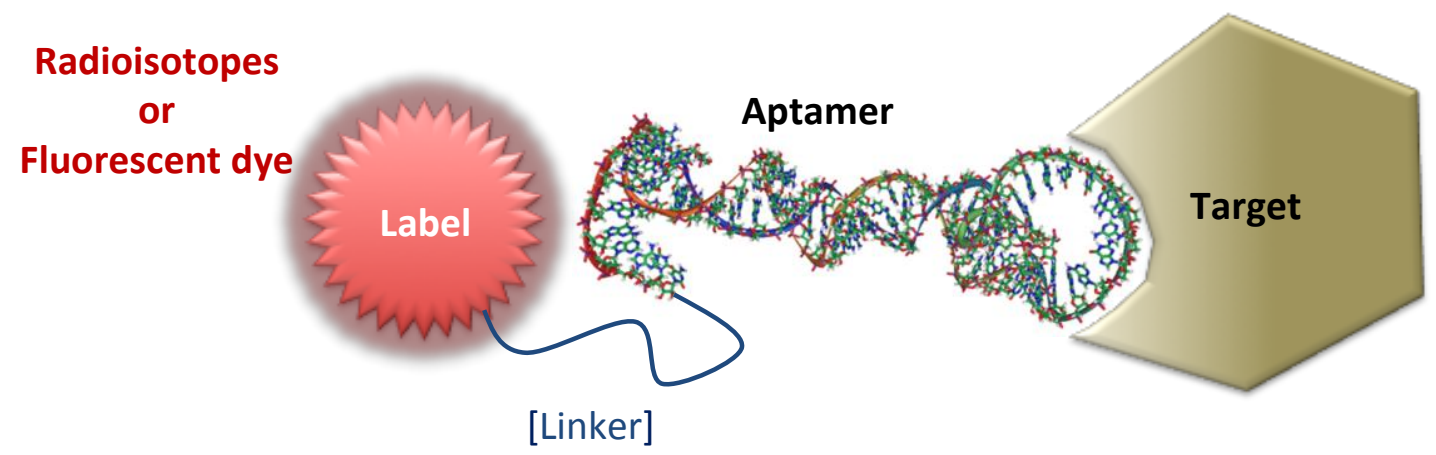

B

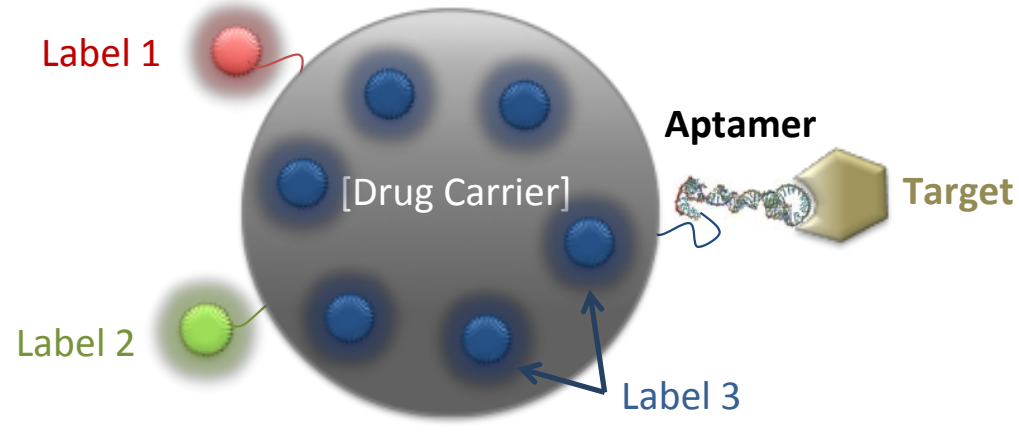


Figure 2
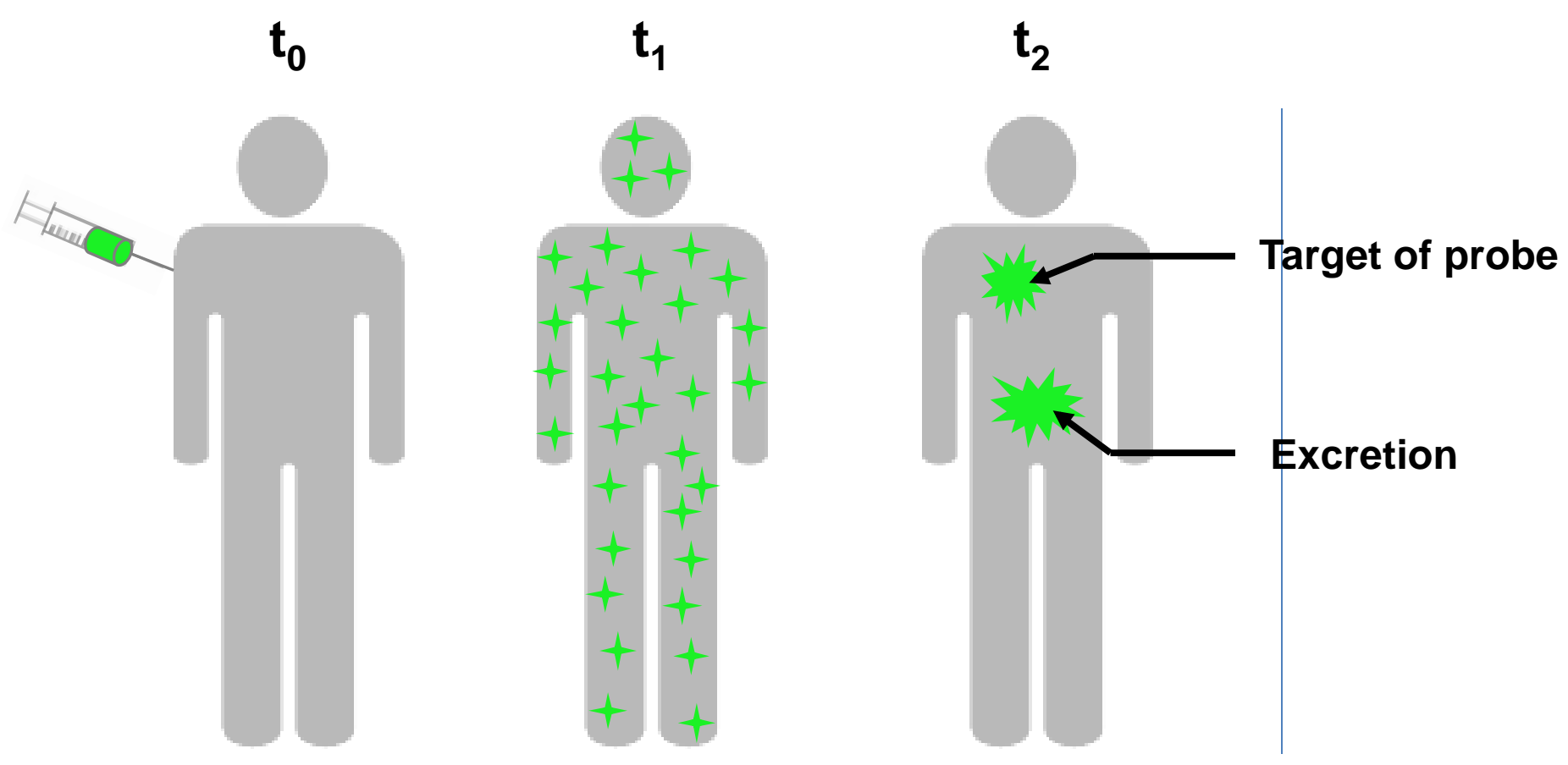


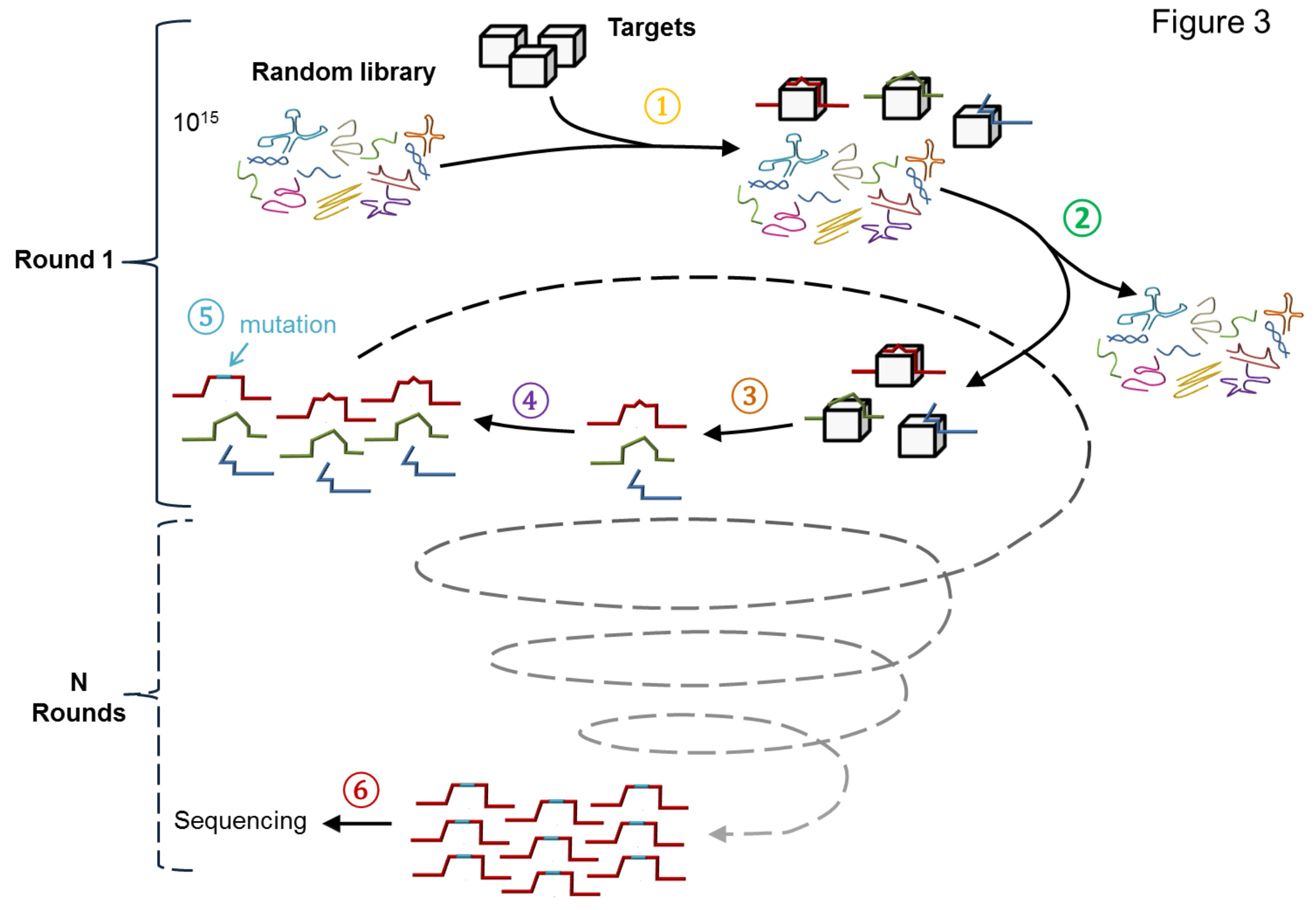


Figure 4

A

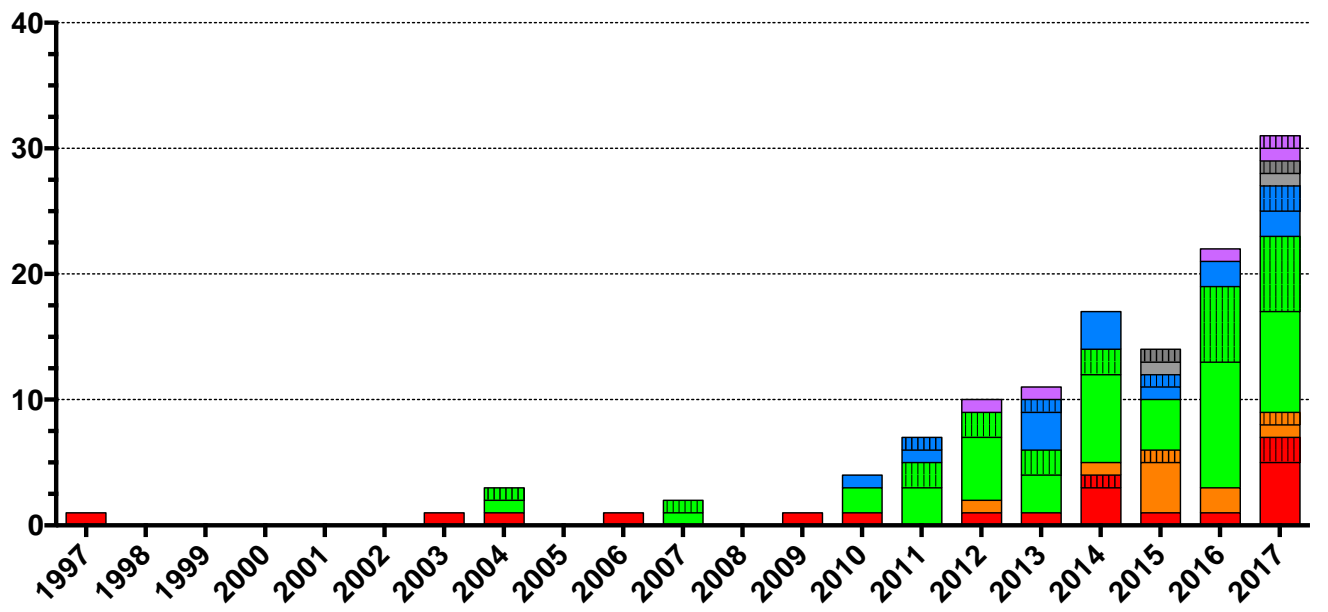

$\square$ SPECT/Scintigraphy $\square$ PET $\square$ Fluorecence $\square$ MRI $\square$ X-ray CT $\square$ Ultrasound 血 Theranostic

B

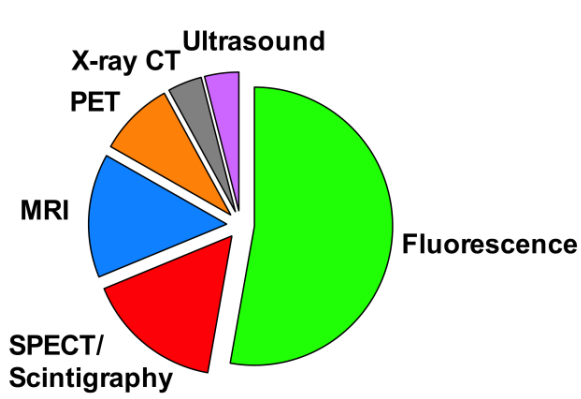

C

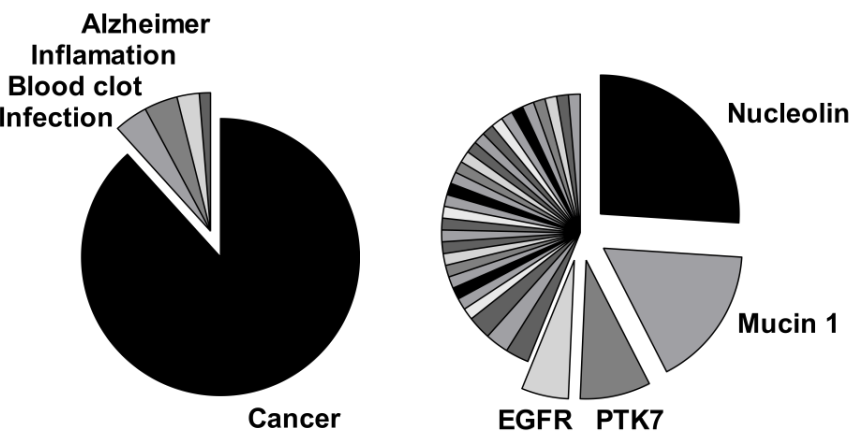

